\title{
Plume structure in high-Rayleigh-number convection
}

\author{
By BABURAJ A. PUTHENVEETTIL ${ }^{1}$ \\ AND JAY WANT H. ARAKERI \\ ${ }^{1}$ Department of Applied Mechanics, Indian Institute of Technology Madras, Chennai, India \\ ${ }^{2}$ Department of Mechanical Engineering, Indian Institute of Science, Bangalore, India \\ jaywant@mecheng.iisc.ernet.in
}

(Received 12 October 2004 and in revised form 29 March 2005)

Near-wall structures in turbulent natural convection at Rayleigh numbers of $10^{10}$ to $10^{11}$ at a Schmidt number of 602 are visualized by a new method of driving the convection across a fine membrane using concentration differences of sodium chloride. The visualizations show the near-wall flow to consist of sheet plumes. A wide variety of large-scale flow cells, scaling with the cross-section dimension, are observed. Multiple large-scale flow cells are seen at aspect ratio $(A R)=0.65$, while only a single circulation cell is detected at $A R=0.435$. The cells (or the mean wind) are driven by plumes coming together to form columns of rising lighter fluid. The wind in turn aligns the sheet plumes along the direction of shear. The mean wind direction is seen to change with time. The near-wall dynamics show plumes initiated at points, which elongate to form sheets and then merge. Increase in Rayleigh number results in a larger number of closely and regularly spaced plumes. The plume spacings show a common log-normal probability distribution function, independent of the Rayleigh number and the aspect ratio. We propose that the near-wall structure is made of laminar natural-convection boundary layers, which become unstable to give rise to sheet plumes, and show that the predictions of a model constructed on this hypothesis match the experiments. Based on these findings, we conclude that in the presence of a mean wind, the local near-wall boundary layers associated with each sheet plume in high-Rayleigh-number turbulent natural convection are likely to be laminar mixed convection type.

\section{Introduction}

Turbulent Rayleigh-Bénard convection, or its variants of natural convection over horizontal surfaces, is extensively studied as a simple system for investigating buoyancy generated turbulence. In addition to the wide engineering applications, turbulent natural convection also occurs in atmospheric boundary layers, oceans and other large water bodies and the Earth's interior. The following non-dimensional parameters characterize turbulent natural convection. Rayleigh number, $R a=g \beta \Delta T H^{3} /(v \alpha)$ is the ratio of the driving buoyancy forces to the restraining dissipative effects; Prandtl number, $\operatorname{Pr}=\nu / \alpha$, a fluid property, is the ratio of the rate of propagation of momentum to that of heat; and aspect ratio, $A R=L / H$, is a geometric parameter. In the case of unsteady Rayleigh-Bénard convection, the ratio of top to bottom fluxes is an additional parameter. The Nusselt number, $N u=q /(\alpha \Delta T / H)$, a non-dimensional flux, depends on the above parameters. Here, $g=$ the acceleration due to gravity, $\beta=$ the coefficient of thermal expansion, $\Delta T=$ the temperature difference between the walls, $H=$ the fluid layer height, $v=$ the kinematic viscosity, $\alpha=$ the thermal diffusivity, 
$L=$ the horizontal dimension of the fluid layer and $q=$ the kinematic heat flux, $Q / \rho C_{p}$ where $Q=$ the heat flux, $-k_{f} \partial T /\left.\partial Y\right|_{y=0}, \rho=$ the fluid density, and $C_{p}=$ the specific heat at constant pressure. If a species is used to create the density difference, then species diffusivity $D$ is used instead of $\alpha$, and Schmidt number $(S c=v / D)$ replaces $P r$. We briefly look at some of the issues of high $R a$ turbulent convection (for details, see the reviews of Spiegel 1971; Adrian, Ferreira \& Boberg 1986; Siggia 1994; Kadanoff 2001).

\subsection{Characteristic scales}

The convection becomes turbulent beyond $R a \sim 10^{5}$ for $P r \sim 7$ (Krishnamurthi 1970), from which point onwards the layer exhibits a clear division into bulk and near wall regions. Dissipative effects are important near the wall while turbulent processes dominate the bulk. The relevant bulk variables are $q, g \beta$ and H. Dimensional analysis implies that the fluctuation scales for velocity, temperature and length in the bulk are (Deardorff 1970),

$$
W_{*} \sim(g \beta q H)^{1 / 3}, \quad \theta_{*} \sim \frac{q}{W_{*}}, \quad Z_{*} \sim H .
$$

Note that $W_{*}$, the Deardorff velocity scale can also be obtained by equating the volume integrated values of kinetic energy dissipation to the buoyant production of kinetic energy.

Diffusive effects are important close to the walls and there is no independent length scale in this region. The relevant independent variables are $\alpha, \nu, g \beta$ and $q$. Based on dimensional reasoning from these variables, Townsend (1959) introduced velocity, temperature and length scales as

$$
W_{o} \sim(g \beta q \alpha)^{1 / 4} \quad \theta_{o} \sim \frac{q}{W_{o}}, \quad Z_{o} \sim \frac{\alpha}{W_{o}} .
$$

Alternatively, taking $\Delta T_{w}$, the temperature difference between the wall and the bulk as the independent variable instead of $q$, Theerthan \& Arakeri (1998) defined near wall velocity, temperature and length scales as

$$
U_{w} \sim\left(g \beta \Delta T_{w}\right)^{1 / 3}(v \alpha)^{1 / 6}, \quad \theta_{w} \sim \Delta T, \quad Z_{w} \sim \frac{\sqrt{\alpha \nu}}{U_{w}} .
$$

It is possible to express the relations between these variables in terms of the nondimensional parameters $R a, N u$ and $P r$. The details of these relations are given in Theerthan \& Arakeri (1998) and Adrian et al. (1986). With an increase in Rayleigh number, it is believed that the bulk starts affecting the near wall flow. This is because the ratio of the bulk to the near-wall velocities increases monotonically with $R a$ as

$$
\begin{gathered}
W_{*} / W_{o} \sim(\mathrm{R} a \mathrm{NuPr})^{1 / 12} . \\
\text { 1.2. Flux scaling }
\end{gathered}
$$

The major focus of work in high $R a$ convection studies has been on the scaling of $N u$ as a function of $R a$. Two asymptotic relations can easily be obtained from dimensional analysis. If the heat transfer is decided solely by the resistance of the boundary layers, the layer height should not be a parameter and we obtain,

$$
N u \sim R a^{1 / 3}, \quad \text { i.e. } q \sim \Delta T^{4 / 3} .
$$

On the other hand, if the heat transfer is decided by the turbulent bulk, and the resistances of the boundary layers are negligible, diffusive properties should not occur 
in the $N u$ relation, and then

$$
N u \sim(R a P r)^{1 / 2} .
$$

The experimentally observed scaling law is $N u \sim R a^{n}$ where $n$ shows a huge variation between 0.20 to 0.382 , with the majority of the exponents being slightly less than 0.3 (Chavanne et al. 2001; Siggia 1994; Niemela et al. 2000; Xia, Lam \& Zhou 2002). The exponent being close to $1 / 3$ implies that the flux is predominantly determined by the near-wall phenomena, but not completely so. This anomalous scaling of $n$ not being equal to $1 / 3$ is expected to be due to a large-scale coherent circulation spanning the two plates which modifies the near-wall boundary layers. As the strength of the mean circulation is expected to be dependent on the geometry of the test section, the circulation also brings in the layer height and the aspect ratio dependence of the flux.

Various theories have been proposed to explain the anomalous flux scaling in highRayleigh-number turbulent natural convection. Each of them involve assumptions about the near-wall phenomena: a near-wall mixing zone (Castaing et al. 1989), a turbulent shear boundary layer (Shraiman \& Siggia 1990) and a Blasius boundary layer (Grossmann \& Lohse 2001). The boundary layers are assumed to span the entire horizontal surface in these theories. In high-Rayleigh-number convection, the nature of near-wall boundary layers and especially their interaction with the large-scale circulation or 'wind' remain a matter of controversy.

\subsection{Near-wall structures}

With the background of these theoretical models, it is worth seeing the experimental and numerical evidence of near-wall structures in turbulent Rayleigh-Bénard convection. The visualizations of near-wall structures have usually been conducted in water $(P r=6)$ up to $R a \sim 10^{9}$. Visualizations at higher Rayleigh number are not available as these studies are conducted under cryogenic conditions. Studies at $R a<10^{8}$, like Spangenberg \& Rowland (1961); Theerthan \& Arakeri (1994, 2000) and Adrian et al. (1986), show randomly moving and merging sheet plumes near the walls. Sheet plumes are buoyant fluid rising in the form of sheets from lines on the horizontal heated surface. Similarly, falling sheets of heavier fluid would be found from the upper surface. These sheets are formed owing to the buoyant boundary layers becoming unstable along the lines. Studies on convection over horizontal heated plates by Sparrow \& Husar (1969) and Kitamura \& Kimura (1995) also show randomly moving sheet plumes in regions away from the edges of the plate. Zocchi, Moses \& Libchaber (1990)'s visualizations in water at $R a \sim 10^{9}$ detected a large-scale flow oriented along the diagonal of the cross-section which was driven by plume columns rising along one corner. They observed near-wall sheet plumes which were swept along the direction of the shear. Funfschilling \& Ahlers (2004) observe sheet plumes with predominant motion along their long axis at $R a \leqslant 10^{9}$, with the direction of this motion shifting about $60^{\circ}$ with a specific frequency for a given $R a$. Near-wall buoyant sheets were also observed in numerical simulations by Kerr (1996) at $R a=2 \times 10^{7}$ at $\operatorname{Pr}=0.7$. Schmidt \& Schumann (1989) find sheet plumes near the bottom surface in large-eddy simulations (LES) of the convective boundary layer at $R a=1.7 \times 10^{16}$.

Measurements with single-point probes give the mean temperature or velocity profiles, but fail to identify the laterally moving and merging sheet plumes. The thermal boundary-layer thickness, defined up to the point where a linear fit to the near-wall temperature profile intersects the well-mixed profile in the bulk, is seen in these studies to scale as $\delta_{t} \sim R a^{-m}$, where $m$ is around 0.3 (e.g. 0.288 by Xin \& Xia 1997; 0.29 by Belmonte, Tilgner \& Libchaber 1994; 0.309 by Chavanne et al. 2001 etc). The boundary-layer thickness measured in this way is seen to depend on the spatial 
location as well as on the direction of traverse (Lui \& Xia 1998). They noticed that there was no repeatability of the measurements until $R a \sim 10^{9}$, beyond which the thermal boundary-layer thickness showed an M-shaped profile in directions perpendicular to the near-wall mean shear direction. Another observation is the presence of the maximum r.m.s. horizontal velocity fluctuations at around the height of the thermal boundary layer (Belmonte et al. 1994). There have also been suggestions that the boundary layer (which is assumed to span the whole width) undergoes a transition at $R a \sim 10^{9}$ by Belmonte et al. (1994) and Chavanne et al. (2001), which has, however, not been observed in the numerical simulations of Verzicco \& Camussi (2003).

Theories based purely on near-wall dynamics have also been proposed for turbulent Rayleigh-Bénard convection. Howard (1964) showed that the near-wall dynamics can be approximated as the periodic growth of a conduction layer which erupts emitting thermals, to obtain the $R a^{1 / 3}$ scaling. Theerthan \& Arakeri (1998) proposed an alternative model based on the experimental observations of persistent sheet plumes and predicted the normalized mean plume spacing $\lambda / Z_{w} \simeq 52$. They also showed that the model predictions of near-wall distributions of mean temperature and r.m.s. of the fluctuations of temperature and vertical velocity match those of experiments.

\subsection{Outer flow}

As seen in (1.4), the bulk velocity increases with increase in $R a$ and is expected to affect the near-wall flow beyond some $R a$. Krishnamurthi and Howard (see Siggia 1994) were the first to report a large-scale circulation or 'wind', which could affect the near-wall boundary layers. More recently, the wind has been the subject of study, mostly in aspect ratio $(A R) \sim 1$ cells. The origin, strength and the effect of this circulation is not known clearly. The exponent in $R e \sim R a^{\gamma}$, where $R e$ is based on mean wind strength and layer height, varies between 0.424 and 0.5 (see the tabulation of Chavanne et al. 2001). This mean circulation has been shown by Burr, Kinzelbach \& Tsinober (2003) and Xia, Sun \& Zhou (2003) to be driven by the mean buoyancy differences in the bulk, and not by the Reynolds stresses. Werne (1993) show from two-dimensional simulations that plumes have an active role in driving the large-scale flow. The sense of this large-scale flow is also noticed to change with time (Niemela et al. 2001). Multiple circulation cells have been found by Verzicco \& Camussi (2003) in low aspect ratio (0.5) cells. Daya \& Ecke (2001) found that, in unity aspect ratio cells, the strength of the temperature and the vertical velocity fluctuations in the bulk, and their scaling with Rayleigh number depended strongly on whether the test section was square or circular in cross-section. Niemela \& Sreenivasan (2003) suggest that the considerable differences (up to $25 \%$ ) in the values of $N u$ for $R a$ between $10^{9}$ and $10^{12}$ could be due to the different time-averaged states of the large-scale flow. Recent particle image velocimetry (PIV) measurements by Xia et al. (2003) show that there is a central low-velocity core where $R e$ scales as $R a^{0.4}$, while in the outer higher-velocity shell, $R e \sim R a^{0.5}$. The circulation is along the diagonal, similar to the observations by Belmonte et al. (1994) and Zocchi et al. (1990). The effect of the largescale flow on the near-wall boundary layers is still a matter of contention. Castaing et al. (1989) assume that the mean wind stabilizes the boundary layers. Shraiman \& Siggia (1990) propose that a turbulent shear boundary layer is formed (similar to the proposal of Kraichnan 1962) and that the heat flux into the diffusive boundary layer is completely carried away by the large-scale flow. Grossman \& Lohse (2000) assume that a Blasius boundary layer spans the cell width and predict the $\mathrm{Ra}$ and $\mathrm{Pr}$ dependence of wind velocity as $R e=0.102 \operatorname{Ra}^{0.447} \mathrm{Pr}^{-0.7 \text { to }-0.73}$ for $10^{8}<R a<10^{10}$ and $3<\operatorname{Pr}<1200$ (Grossman \& Lohse 2002). 


\subsection{Prandt-number dependence}

The Prandtl-number dependence of the Nusselt number in the high $R a$-high $P r$ regime is not conclusive as there have been very few studies. At a $\operatorname{Pr}$ of 2750, Goldstein, Chiang \& See (1990) obtained $N u=0.0659 R a^{1 / 3}$. Ashkenazi \& Steinberg (1999) obtained a fit of $N u=0.22 R a^{0.3 \pm 0.03} \mathrm{Pr}^{-0.20 \pm 04}$ for $10^{9}<R a<10^{14}$ and $1<\operatorname{Pr}<93$. The errors in their exponent do not allow rigorous conclusions about the scaling. Xia, Lam \& Zhou (2002) covered a wide Prandtl-number range of $4<P r<1350$ for $10^{7}<R a<10^{10}$ (for $\operatorname{Pr}>396, R a$ was limited to less than $10^{9}$ ) and obtained a weak $\operatorname{Pr}$ dependence, $N u=0.14 R a^{-0.297} \mathrm{Pr}^{-0.03}$. In the range of $0.022<\operatorname{Pr}<15$ at $\operatorname{Ra} \leqslant 10^{7}$, $\mathrm{Nu}$ showed negligible dependence on $\operatorname{Pr}$ for $\operatorname{Pr}>0.35$ (Verzicco \& Camussi 1999). A similar weak decrease of $N u$ for $0.7<P r<7$ in the range $10^{4}<R a<10^{7}$ was observed by Kerr \& Herring (2000).

The large-scale flow strength is generally seen to decrease with $\operatorname{Pr}: \operatorname{Re}=$ $0.335 \mathrm{Ra}^{0.495} \mathrm{Pr}^{-0.88}\left(\operatorname{Re}=1.09 \mathrm{Ra}^{0.43} \mathrm{Pr}^{-0.76}\right.$ based on oscillation frequency) for $10^{6}<$ $R a<10^{11}, 6<P r<1027$ (Lam et al. 2002) and $\operatorname{Re}=2.6 \operatorname{Ra}^{0.43 \pm 0.02} \operatorname{Pr}^{-0.75 \pm 0.02}$ (Ashkenazi \& Steinberg 1999). Verzicco \& Camussi (1999) observed that with increase in $\mathrm{Pr}$, the large-scale flow strength reduces, but still determines the structure of the boundary layer. At larger $P r$, the mean wind played a negligible role in heat transport, which was mainly conducted by plumes. Larger and fewer plumes were observed by Kerr \& Herring (2000) with increasing Pr, with the network-type near-wall structure changing to clear cells.

In the high Rayleigh-number regime $\left(R a>10^{7}\right)$, Prandtl-number dependence of the Nusselt number for $\operatorname{Pr}>1$ is much weaker than the $-1 / 7$ power predicted by the theories of Castaing et al. (1989) and Shraiman \& Siggia (1990). At very high Pr, the viscous boundary layers reach their limiting thickness as the large-scale flow strength reduces (Grossmann \& Lohse 2001). Owing to the thicker viscous boundary layer, the effects of the large-scale flow shear would be felt less by the thermal boundary layer at higher $\mathrm{Pr}$. Plumes start to play a major role in the heat transport. Hence, at high $\mathrm{Pr}, \mathrm{Nu}$ is expected to become independent of $\mathrm{Pr}$ and to follow the classical $R a^{1 / 3}$ scaling. A recent theory of P. Constantin and C. Doering (see Chavanne et al. 2001) predicts a $N u \sim R a^{1 / 3}$ bound for infinite-Prandtl-number convection.

\subsection{Present study}

From the current state of understanding, the nature of near-wall boundary layers in high-Rayleigh-number turbulent Rayleigh-Bénard convection is not clear. The origin of large-scale flow is also not well-established. The interaction of the large-scale flow with the boundary layers is even less clear. Most of the experiments at $R a>10^{9}$ have been under cryogenic conditions and thus visualizations have not been possible. The aim of the present study was to investigate high-Rayleigh-number turbulent natural convection in the high-Prandtl-number regime, focusing on the nature and the role of near-wall coherent structures. We achieve this objective by setting up unsteady turbulent natural convection, driven by density differences across a thin permeable horizontal partition separating two tanks of square planform cross-sections. The gravitational potential due to a heavier fluid (brine) above a lighter fluid (water) across the partition drives the flow, which is resisted by the presence of the micro porous partition. At low pore sizes in the membrane, the transport across the partition would become diffusion dominated, while the transport above and below the partition becomes similar to turbulent natural convection above flat horizontal surfaces. The concentration difference between the membrane surface and the bulk is equivalent to the temperature difference between the wall and the bulk in Rayleigh-Bénard 
convection. As molecular diffusivity of $\mathrm{NaCl}$ in water is about 100 times lower than the thermal diffusivity, large values of the Rayleigh number and the Schmidt number are achieved through this arrangement. We achieve $R a \sim 10^{11}$ at $S c \sim 602$. The main advantages with the present experimental technique are that high $R a$ and high $S c$ values are achieved without going in for very large set-ups and the near-wall structure of convection is easily visualized (Puthenveettil 2004).

The paper is organized as follows. The experimental set-up and the measurement details are discussed in $\S 2$. We first show in $\S 3$ that the flux scaling obtained is similar to those achieved in other high-Rayleigh-number Rayleigh-Bénard convection experiments at comparable Prandtl numbers. The images of the near-wall plume structure, which show merging sheet plumes, are discussed in $\S 4$ and 5 to form conclusions about the nature and origin of the large-scale flow, near-wall plume dynamics, as well as about the interaction of the large-scale flow with the near-wall plumes. We hypothesize that the near-wall boundary layers associated with the plumes are most likely to be laminar natural convection boundary layers, which become unstable to give rise to the sheet plumes near the wall. This assumption is validated in $\S 7.1$ by showing that the predictions of a model constructed on this hypothesis reproduce the measured mean spacings between the near-wall sheet plumes. Based on this finding, we conclude in $\S 8$ that the near-wall boundary layers in high-Rayleigh-number turbulent free convection, in the presence of a large-scale circulation, are laminar natural-convection boundary layers, forced externally by the mean shear.

\section{Experimental set-up and data analysis}

\subsection{Set-up}

The experimental set-up (figure 1) consists of two glass compartments of square crosssection, arranged one on top of the other with a fine membrane fixed horizontally in between them. The membrane used is Pall Gellmann ${ }^{\mathrm{TM}}$ NX29325 membrane disk filter having a random pore structure with specifications of mean pore size $0.45 \mu \mathrm{m}$ and mean thickness $l_{m}=142.24 \mu \mathrm{m}$. Figure 2 shows the $3000 \times$ scanning electrode microscope (SEM) image of the membrane surface. The open area factor of the membrane is estimated as $\Gamma=0.6$ by calculating the occupied area from a binary image. The binary image is obtained by choosing an appropriate threshold so that the features match the original image shown in figure 2 . The membrane is slightly stretched before fixing to keep it taut during the experiment. The bottom tank is filled with distilled water tagged with a small amount of sodium fluoresceine, and then the top tank is filled with brine. A thin-walled tank with a thin glass plate on top of a sponge bottom, and just fitting into the top compartment, is kept within the top compartment during the filling process; this prevents undue initial mixing due to direct impingement of brine on the membrane. Once the brine in the top tank reaches the height of the bottom tank, this inner tank is slowly removed to initiate the experiment. A thin transparent Plexiglas sheet of dimensions of the test-section crosssection is kept floating on the top of the brine solution to prevent evaporation and to produce similar boundary conditions in the two compartments. The whole assembly is mounted on a levelling table to make the membrane horizontal. Diffusion of salt across the membrane sets up unstable layers on either side and causes convection in the two tanks. It is a run-down experiment; convection eventually stops after density equalization in the tanks, which typically takes about 2 days.

A horizontal laser light sheet, expanded and collimated from a $5 \mathrm{~W}$ Spectra Physics Ar-ion laser Stabilite ${ }^{\mathrm{TM}} 2017$ is passed just above $(<1 \mathrm{~mm})$ the membrane to visualize the planform view of the near-wall structures. The laser sheet could be rotated $90^{\circ}$ by 
(a)

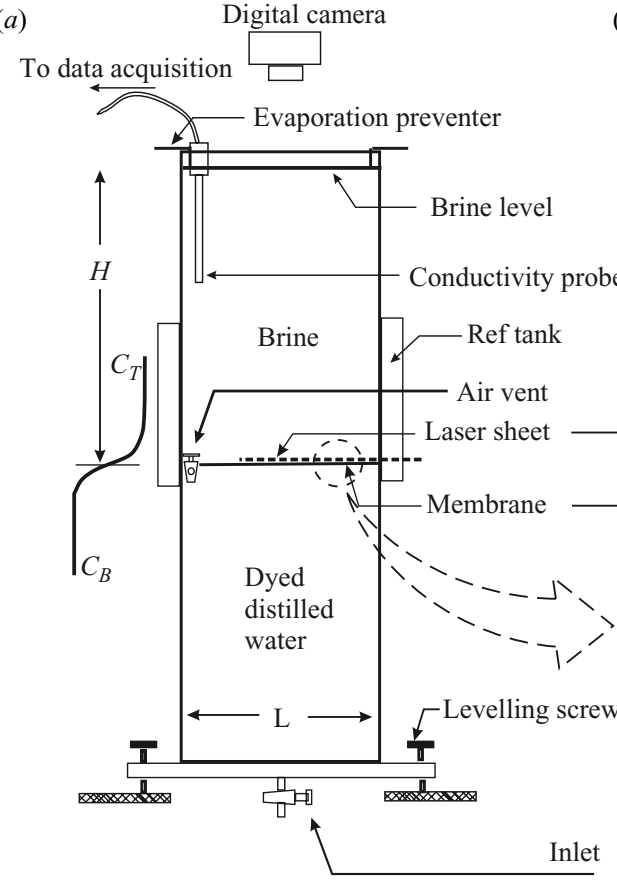

(b)

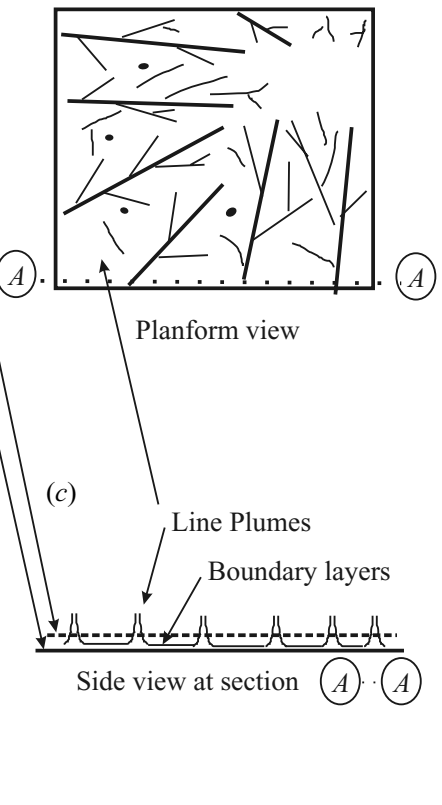

FIGURE 1. Experimental set-up and the visualization process. (a) The overall set-up. (b) The planform view of the plume structure just above the membrane, as seen from the top, at the intersection of a horizontal laser sheet with the ascending dyed lighter fluid. (c) The side view in a vertical plane shows rising sheet plumes.

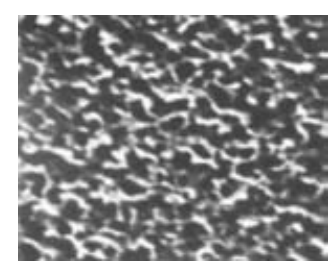

Figure 2. SEM image $(3000 \times)$ of the Pall Gellman ${ }^{\mathrm{TM}}$ membrane.

rotating the beam expander lens so as to view the structures in a vertical plane. The laser sheet could also be moved horizontally and vertically. The dye in the bottom solution while convecting upward fluoresces on incidence of the laser beam to make the plume structure visible. The diffusion coefficient of salt is about three times the diffusion coefficient of dye $(S c=2000$ for the dye as against $S c=602$ for the salt). The very low concentration (1.2 p.p.m.) of the dye used causes negligible density change and hence the dye can be considered as a passive tracer. A visible long-passfilter glass, Coherent optics OG-515, is used to block any scattered laser light and to allow the emitted fluorescence to pass through. The images are captured on a digital handycam Sony DCR PC9E. Experiments are conducted in two set-ups, one having a $15 \mathrm{~cm} \times 15 \mathrm{~cm}$ cross-section and the other a $10 \mathrm{~cm} \times 10 \mathrm{~cm}$ cross-section. In both the set-ups, the top and bottom compartments are $23 \mathrm{~cm}$ high. Thus we have two aspect ratio values, 0.65 and 0.435 . We use starting top tank concentrations of $10 \mathrm{~g}^{-1}$ and $3 \mathrm{~g}^{-1}$ to study the plume structure under different Rayleigh numbers. The experimental set-up and the procedure are discussed in detail in Puthenveettil (2004). 


$\begin{array}{cccc}\text { Test-section cross-section }(\mathrm{cm}) & H(\mathrm{~cm}) & A R & C_{T}^{0}\left(\mathrm{~g}^{-1}\right) \\ 15 \times 15 & 23 & 0.65 & 10 \\ & & & 3 \\ 10 \times 10 & 23 & 0.435 & 10\end{array}$

TABLE 1. Experimental parameters.

The flux is estimated from the transient measurement of the top tank concentration. The concentration of sodium chloride $(\mathrm{NaCl})$ in the top tank is obtained from the measurement of electrolytic conductivity by an ORION SENSORLINK ${ }^{\mathrm{TM}}$ PCM100 conductivity measurement system (ORION 1999a, $b$ ). The cell has an outer diameter of $12 \mathrm{~mm}$ and $0.5 \%$ full-scale accuracy in the present measuring range of $2 \mathrm{mS}$ $20 \mathrm{mS}$, with automatic temperature compensation. The probe is calibrated for cell constant before each experiment, and the measured conductivities converted to $\mathrm{NaCl}$ concentrations using the standard relation from Lide (2001). Before each experiment, as a check against the drift of the probe, known $\mathrm{NaCl}$ concentrations made from prepared samples and dilution samples from the top tank brine solution are measured and compared.

\subsection{Data analysis}

We assume that the fluid in both the compartments is well mixed in the region away from the membrane, and hence can be represented by the top tank concentration $C_{T}$ and the bottom tank concentration $C_{B}$. This assumption is valid for high-Rayleighnumber convection which has thin boundary layers and a well-mixed bulk. We checked this assumption by quantitative planar laser induced fluorescence (QPLIF) and by traversing the probe in the bulk. Using mass balance, at any instant of time $t$, the concentration difference between the tanks, $\Delta C(t)$ is,

$$
\Delta C(t)=C_{T}(t)-C_{B}(t)=\left(1+\frac{V_{T}}{V_{B}}\right) C_{T}(t)-C_{T}^{0} \frac{V_{T}}{V_{B}},
$$

where $V_{T}$ is the top tank solution volume, $V_{B}$ the bottom tank solution volume and the superscript ${ }^{0}$ indicates the initial values. The rate of change of the well-mixed top tank concentration would give the flux of $\mathrm{NaCl}$ into the top tank at any instant as,

$$
q(t)=-H \frac{\mathrm{d} C_{T}(t)}{\mathrm{d} t},
$$

where $H$ is the top and the bottom liquid-layer heights. This is the mass transfer rate per unit area of the tank cross-section. We make transient measurement of the changing well-mixed concentration in the top tank to estimate $\Delta C$ from (2.1) and the flux from (2.2). An exponential decay fit of the form

$$
C_{T}(t)=y_{0}+A_{1} \exp \left(-t / b_{1}\right)+A_{2} \exp \left(-t / b_{2}\right)+A_{3} \exp \left(-t / b_{3}\right)
$$

through the $C_{T}$ vs. $t$ curve is used to calculate $\mathrm{d} C_{T} / \mathrm{d} t$. In most experiments, concentration measurement and visualizations are conducted simultaneously. The quantity of dye is chosen so that the fluorescence intensity is enough for sufficient visibility while it is low enough not to affect the measured conductivity. The major parameters that are changed in the experiments are the aspect ratio and the starting concentration; the values of these parameters are shown in table 1 . The low-concentration experiment is 


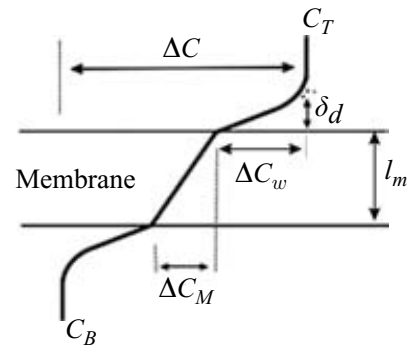

FIGURE 3. Concentration profile across the membrane $\left(\Delta C_{M}\right)$ and the boundary layer $\left(\Delta C_{w}\right)$.

conducted to visualize the near-wall structures at lower driving potentials (i.e lower $R a$ ) as well as to see whether the flux is independent of the starting concentration.

It should be noted that convection in the present study is unsteady non-penetrating convection (UNP) while most of the investigations in the literature are for steady Rayleigh-Bénard convection (RBC). The top surface in the present study is adiabatic and the driving concentration difference and flux keeps changing throughout the experiment. Adrian et al. (1986) have shown that for constant flux, UNP and RBC are similar with respect to turbulence characteristics. In the present case, even though the flux is not constant, the situation can be considered as quasi-steady as the time scale of change of concentration $(\Delta C /(\mathrm{d} \Delta C / \mathrm{d} t))$ is much larger (10 times) than the time scale of one large-scale circulation $\left(H / W_{*}\right)$.

\section{Flux scaling}

Diffusion transport across the membrane results in a concentration drop of $\Delta C_{M}$ across the membrane thickness (figure 3). We estimate this concentration drop from the relation $\Delta C_{M}=q l_{m} / \Gamma D$ where $D=$ the molecular diffusivity of $\mathrm{NaCl}$. The effective concentration difference on one side of the partition is $\Delta C_{w}=\left(\Delta C-\Delta C_{m}\right) / 2$. We use $\Delta C_{w}$ as the relevant driving potential and

$$
R a_{w}=g \beta \Delta C_{w} H^{3} / \nu D
$$

as the relevant Rayleigh number for comparison with the high-Rayleigh-number turbulent natural convection results in the literature.

Theerthan \& Arakeri (2000) have shown that in turbulent free convection, normalizing the flux with near-wall variables is more appropriate than normalizing with the total temperature difference between the plates and the layer height, as is done in $N u$. The new representation can be written as

$$
R a_{\delta}^{-1 / 3}=\frac{q}{D \Delta C_{w} / Z_{w}}
$$

where $Z_{w}=\left(v D /\left(g \beta \Delta C_{w}\right)\right)^{1 / 3}$ is the near-wall length scale for turbulent free convection given by (1.3). Here, $R a_{\delta}$ is the Rayleigh number based on the diffusion layer thickness, $\delta_{d} ; \delta_{d}=D \Delta C_{w} / q$ in the present case. This representation does not have the layer height as a parameter, and hence is a better representation in turbulent free convection where the near-wall phenomena decides the flux; the differences in the near-wall phenomena are reflected better in the variation of $R a_{\delta}^{-1 / 3}$ than that of $N u$. $R a_{\delta}^{-1 / 3}$ is a direct measure of heat flux and varies only between 0.1 and 0.3 for various types of convection, $A R, R a$ and $P r$. A correlation $N u=C R a^{n}$ for RBC, rewritten in terms of $R a_{\delta}^{-1 / 3}$ becomes $R a_{\delta}^{-1 / 3}=A R a^{n-1 / 3}$, where $A=2^{4 / 3} C . R a_{\delta}^{-1 / 3}$ is a constant 


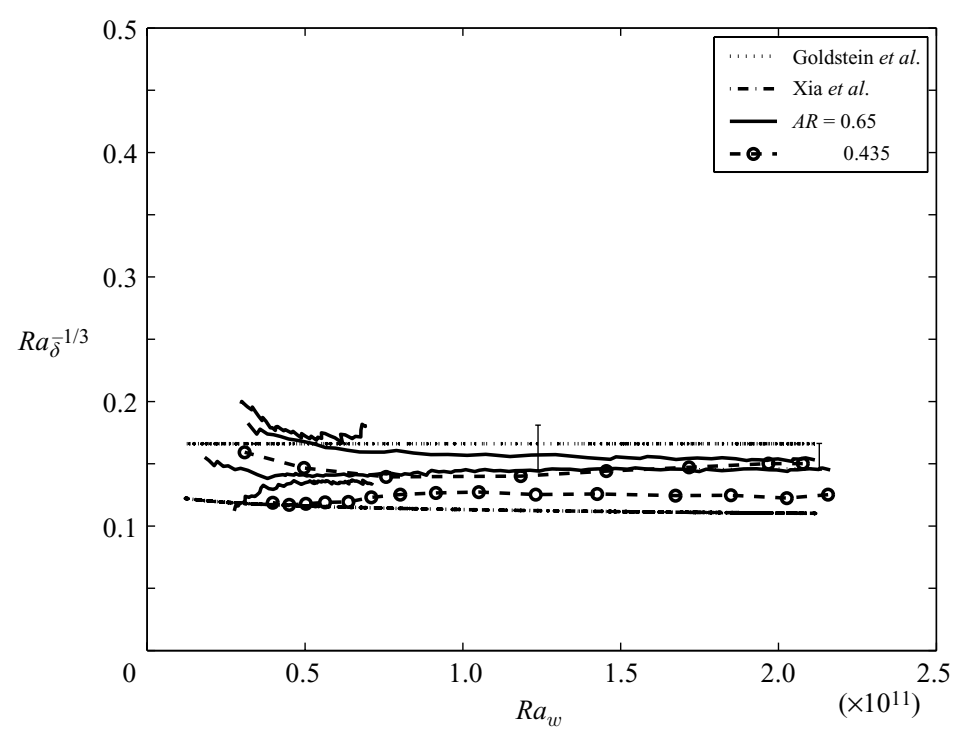

FIGURE 4. Variation of the non-dimensional flux, $R a_{\delta}^{-1 / 3}$, with the Rayleigh number for different $A R$ and $C_{T}^{o}$.

if flux scales as $\Delta C^{4 / 3}$, i.e. if $N u \sim R a^{1 / 3}$; if $n=2 / 7$ then $R a_{\delta}^{-1 / 3}=A R a^{-1 / 21}$, a weak function of $R a$. A similar feature is observed in wall-bounded turbulent shear flows where variations of the coefficient of friction $C_{f}$ with Reynolds number are found to be small. In fact, $R a_{\delta}^{-1 / 3}$ is analogous to the $C_{f}$ representation of wall shear stress.

Figure 4 shows the variation of $R a_{\delta}^{-1 / 3}$ with $R a_{w}$. The plot includes the results of experiments with a starting concentration of $C_{T}^{0}=10 \mathrm{gl} l^{-1}$ at the two aspect ratios of 0.65 and 0.435 , as well as experiments with starting concentration of $C_{T}^{0}=3 \mathrm{~g}^{-1}$ for $A R=0.65$. Repeated experiments are also shown to indicate the variability of the present results. The error bars shown in the plot are for the $A R=0.65$ experiment. The line $R a_{\delta}^{-1 / 3}=0.166$ shown in the figure is obtained from the correlation of Goldstein et al. (1990), $N u=0.065 R a^{1 / 3}$ for $10^{8} \leqslant R a \leqslant 10^{12}$ and $0.86 \leqslant A R \leqslant 24$ at $P r=2750$. The value of $R a_{\delta}^{-1 / 3}$ for Xia et al. (2002) is also shown in the figure. Xia obtained $N u=0.14 R a^{0.297} \mathrm{Pr}^{-0.03}$ for $10^{7} \leqslant R a \leqslant 10^{11}$ for $4 \leqslant \operatorname{Pr} \leqslant 1352$. This relation is an adjusted fit over the whole $\operatorname{Pr}$ range; the measurements for $\operatorname{Pr}>556$ are only for $R a \leqslant 10^{8}$ and the $\operatorname{Pr}$ dependence seems to vary for different $R a$ ranges (see figure 2 of Xia et al.).

The results of Goldstein et al. and Xia et al. envelope the present experimental results. The deviation at the left-hand end of each curve cannot be inferred to be a change in the flux scaling, as the errors involved in calculating $\Delta C_{w}$ become large when the concentration differences between the tanks tend to zero. The present experimental values of $R a_{\delta}^{-1 / 3}$ are nearly constant, implying that the flux scales very nearly as the $4 / 3 \mathrm{rd}$ power of $\Delta C_{w}$. This is intuitively understandable as the effect of large-scale coherent circulation on the near-wall boundary layers is expected to become weaker with increase in Prandtl number (see $\S 1.5$ ).

The low $R a_{w}$ range of the present experiments, does not allow clear identification of the exponent in the scaling law vis-à-vis that of Goldstein or Xia. Both the exponents look probable within the present range of $R a_{w}$. The present experimental results at $S c=602$ are closer (about $15 \%$ lower at $R a_{w} \sim 10^{11}$ ) to the values in Goldstein 


\begin{tabular}{rccccccc}
\hline Exp. & Figure & $\Delta C_{w}\left(\mathrm{~g}^{-1}\right)$ & $R a_{w}$ & Flux $\left(\mathrm{mg} \mathrm{cm}^{-2} \mathrm{~min}^{-1}\right)$ & $R a_{q}$ & $W_{*}\left(\mathrm{~cm} \mathrm{~s}^{-1}\right)$ & Time (min) \\
10jan & $9(a)$ & 3.26 & $2.07 \times 10^{11}$ & 0.1147 & $1.89 \times 10^{14}$ & 0.3123 & 18.17 \\
10jan & 8 & 3.21 & $2.04 \times 10^{11}$ & 0.1116 & $1.83 \times 10^{14}$ & 0.31 & 40.67 \\
10jan & $9(b)$ & 2.94 & $1.87 \times 10^{11}$ & 0.0997 & $1.64 \times 10^{14}$ & 0.298 & 132.17 \\
10jan & $9(c)$ & 2.65 & $1.69 \times 10^{11}$ & 0.088 & $1.49 \times 10^{14}$ & 0.289 & 237.67 \\
4feb & $9(d)$ & 2.65 & $1.67 \times 10^{11}$ & 0.09 & $1.49 \times 10^{14}$ & 0.289 & 211.5 \\
31jan & $5(a)$ & 1.0 & $6.39 \times 10^{10}$ & 0.0209 & $3.43 \times 10^{13}$ & 0.177 & 172.5
\end{tabular}

TABLE 2. Parameters corresponding to planform structures for $A R=0.65$. The time corresponds to that from the initiation of each experiment.

et al.'s (1990) experiments, which are at $S c=2750$; the effect of $S c$ on flux seems to be weak at high $S c$. The experimental results seem to be independent of the starting concentration. The aspect ratio dependence on the flux is weak and is not distinguishable within the variability of the present experimental results for the low range of $A R$. Still, the flux at lower $A R$ seems to be lower than at higher $A R$. This is similar to the results of Wu \& Libchaber (1992) who find decreasing flux with decreasing $A R$ for $A R \leqslant 1$. The present variability in flux is about $25 \%$ at $R a_{w} \sim 10^{11}$. A large variability $(\sim 25 \%)$ of $N u$ is present in the results of previous investigators for $10^{9} \leqslant R a \leqslant 10^{12}$, as noted in the review of Niemela \& Sreenivasan (2003) (see their figure 5). They hypothesize the presence of different time-averaged mean flow for a given set of conditions as the possible cause of this variation. It is also possible that the variation of the present experimental results is due to the slight changes in the open area factor of the membrane. These issues cannot be resolved within the limitations of the present experimental effort. Therefore, we conclude that at Rayleigh numbers of $10^{10}-10^{11}$ and a Schmidt number of 602, the flux from the present experiments scale similarly to that in turbulent Rayleigh-Bénard convection $\left(N u \sim R a^{1 / 3}\right)$ at high $P r$.

\section{Plume structure at $A R=0.65$}

$$
\text { 4.1. } R a_{w} \sim 10^{10}
$$

Figure 5(a) shows the planform plume structure, obtained when the upcoming dyed fluid from the bottom tank intersects the horizontal laser light sheet, which is typically less than $1 \mathrm{~mm}$ above the membrane. The experiment was started with an initial top tank concentration of $C_{T}^{o}=3 \mathrm{~g}^{-1}$. The image shows the full test-section cross-section of $15 \mathrm{~cm} \times 15 \mathrm{~cm}$ and is at $R a_{w}=6.39 \times 10^{10}$. The other parameter details are shown in table 2. The white lines in the image are the top view of the dyed lighter fluid rising from the membrane surface, and represent bases of sheet plumes. Figures $5(b)$ to $5(d)$ show the planform views at $2 \mathrm{~cm}, 4 \mathrm{~cm}$ and $6 \mathrm{~cm}$ above the membrane surface. These images were obtained by placing the horizontal laser sheet at these heights. The time period between figures $5(a)$ and $5(d)$ is 6 min so that $R a_{w}$ is nearly the same in all the cases shown. The side view of the plume structure in a vertical plane $2 \mathrm{~cm}$ from the sidewall (i.e. $2 \mathrm{~cm}$ above the bottom edge of figure $5 a$ ) after $10 \mathrm{~min}$ of figure $5(d)$ is shown in figure $5(e)$.

\subsubsection{Large-scale flow dynamics}

The near-wall planform of figure 5(a) shows aligned sheet plumes near the leftand the right-hand walls. There is a region in the centre which does not have aligned structures. The planform views at different heights show cross-sections of a rising 


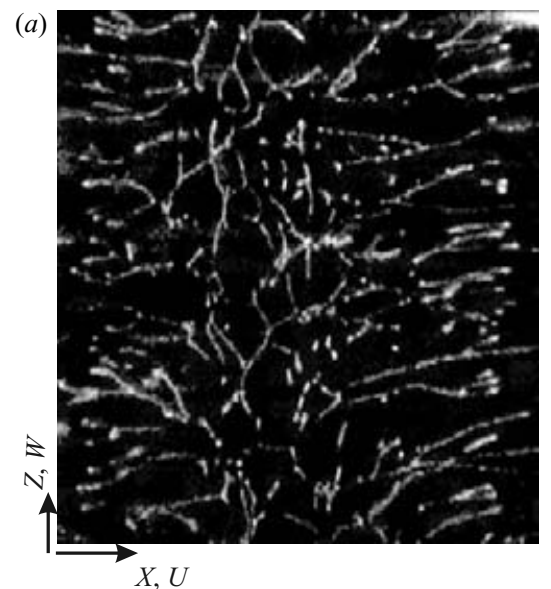

(d)

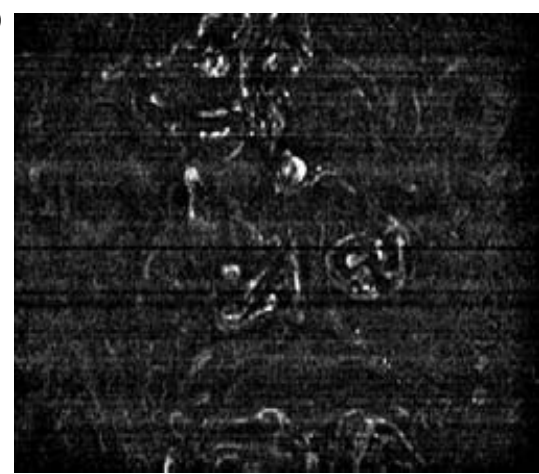

(b)

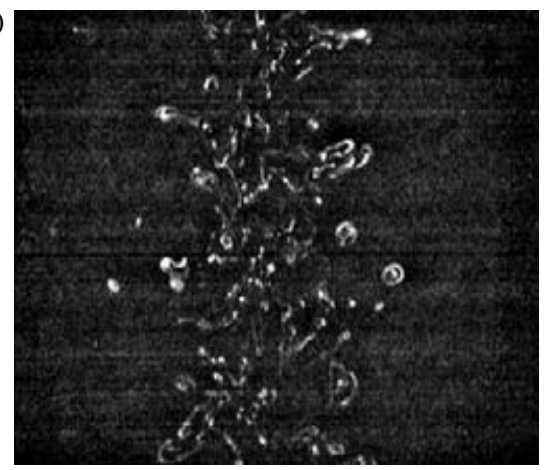

(e)

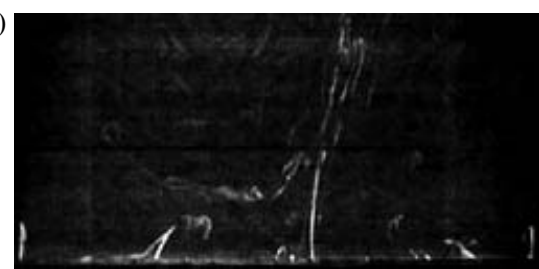

(f)

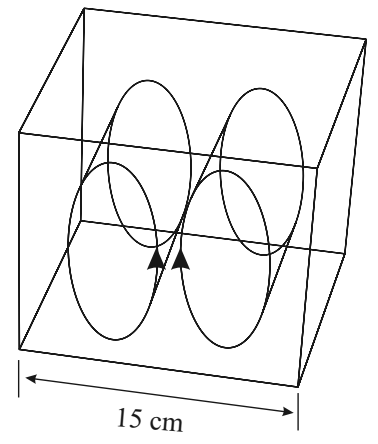

(c)

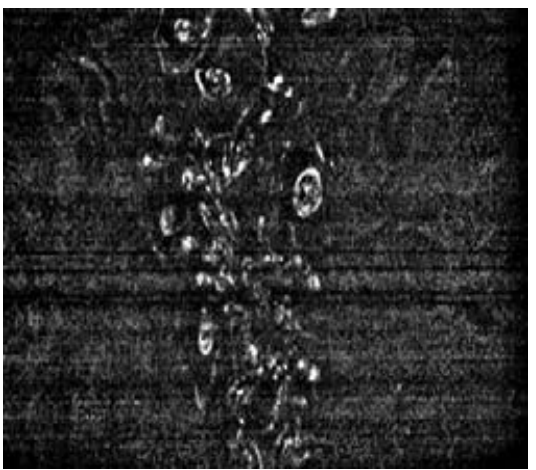

FIGURE 5. $(a)-(d)$ The sequence of planform images for $A R=0.65$ and $C_{T}^{o}=3 \mathrm{~g} 1^{-1}\left(\mathrm{Expt}^{\mathrm{p}}\right.$ 31 jan) at different heights for $R a_{w}=6.39 \times 10^{10}$. The parameter details of $(a)$ are shown in table 2. (a) Grazing. (b) $2 \mathrm{~cm}$. (c) $4 \mathrm{~cm}$. (d) $6 \mathrm{~cm}$. (e) Side view of plume structure in a vertical plane. $(f)$ Diagram of the infered large-scale flow.

column of plumes. Note that sheet plumes are seen only very close to the wall; at a short height from the wall, they turn into axisymmetric plumes with mushroom-like caps, and merge. The intersections of these axisymmetric plumes with the laser sheet are seen as points or circles in figures $5(b)$ and $5(c)$. The plumes loose their identity owing to mixing at a height of $6 \mathrm{~cm}$ from the membrane. The rising column in the 
centre, with small near-wall plumes being swept towards it is clearly seen in the side view of figure $5(e)$.

Based on these observations, we infer the large-scale flow to be two counter-rotating rolls, schematically shown in figure $5(f)$. The large-scale flow is driven by the rising plume column in the centre. This rising line of lighter fluid in the centre creates regions of fluid which are coming down near the wall, to create the two counterrotating large-scale flow cells. The large-scale flow cells in turn create a mean shear near the membrane, aligning the near-wall plumes and sweeping the smaller nearwall plumes into the main rising plume column. The planform plume structure in figure $5(a)$ is the result of this larger mean shear near the walls with a rising column in the centre. Hence, the effect of the large-scale flow on the near-wall structures in high Rayleigh-number turbulent free convection is to align the near-wall sheet plumes in the direction of the mean shear. This is an important result of the present study, which helps us later in making inferences about the nature of the boundary layers in between the sheet plumes in the presence of a mean wind.

Using a spatial intensity correlation technique (Gendrich \& Koochesfahani 1996) we estimate the plume column velocities. The maximum plume column rise velocities are $V_{L S}=0.16 \mathrm{~cm} \mathrm{~s}^{-1}(R e=412.1)$ at $R a_{w}=6.23 \times 10^{10}$, where the subscript ${ }_{L S}$ denote large-scale. There are downflow regions with velocity of about $V_{L S}=0.02 \mathrm{~cm} \mathrm{~s}^{-1}$ $(R e=52.5)$ near the membrane on both sides of the rising plume column. The mean time of circulation $\left(H / V_{L S}\right)$ of the large-scale flow cell is about $4.25 \mathrm{~min}$. We now briefly compare the earlier estimates of large-scale flow velocity with the present measurements. The high-Prandtl-number measurements of large-scale flow velocity by Lam et al. (2002) (see $\S 1.5)$ for the present $R a_{w}$ and $S c$ give $R e=264.5\left(V_{L S}=0.103 \mathrm{~cm} \mathrm{~s}^{-1}\right)$. Ashkenazi \& Steinberg (1999) correlation (\$1.5) shows a possible variation of $501.9<$ $R e<1752.1\left(0.195 \mathrm{~cm} \mathrm{~s}^{-1}<V_{L S}<0.68 \mathrm{~cm} \mathrm{~s}^{-1}\right)$ for the present $R a_{w}-S c$ combination. Niemela et al.'s (2001) measurements of mean wind velocity gives $P e=0.13 R a^{0.5}$, which for the present parameter values gives $V_{L S}=0.02 \mathrm{~cm} \mathrm{~s}^{-1}$, similar to the present downflow velocities. The present measurements of plume column rise velocities are of the same order as the earlier estimates of large-scale flow velocity, confirming the finding that large-scale flow is driven by plume columns.

We can also estimate the mean velocity of the large-scale circulation from the bulk velocity scale of (1.1), using the constant value of $R a_{\delta}^{-1 / 3}$ noted in $\S 3$. If $R a_{\delta}^{-1 / 3}=k_{1}$, equivalent to $N u \sim R a^{1 / 3}$, and if the large-scale flow velocity $V_{L S}=k_{2} W_{*}$ (where $k_{1} \sim 0.166$ and $k_{2} \sim 1$ ), we obtain

$$
V_{L S}=k_{1}^{1 / 3} k_{2}(D / S c)^{1 / 9}\left[g \beta \Delta C_{w} H^{3 / 4}\right]^{4 / 9} .
$$

The Reynolds number from (4.1),

$$
R e=\frac{k_{1}^{1 / 3} k_{2}}{2^{4 / 9}} R a^{0.44} S c^{-2 / 3},
$$

with the prefactor 0.4, matches the $R a$ and $P r$ dependence of Lam et al. (2002) and Grossman \& Lohse (2002) ( $§ 1.4$ and 1.5). A value of $n=2 / 7$ in the $N u \sim R a^{n}$ scaling gives $R e \sim R a^{0.43}$. Further, the Deardorff velocity scale for figure $5(e)$ is $0.18 \mathrm{~cm} \mathrm{~s}^{-1}$; the same order as the plume column rise velocity. Hence, the Deardorff velocity scale seems to be an appropriate estimate of the mean large-scale flow velocity and correctly predicts the Prandtl number dependence. The low Reynolds number of the large-scale flow rules out the possibility of a near-wall turbulent shear boundary layer created by the large-scale circulation. 
(a)
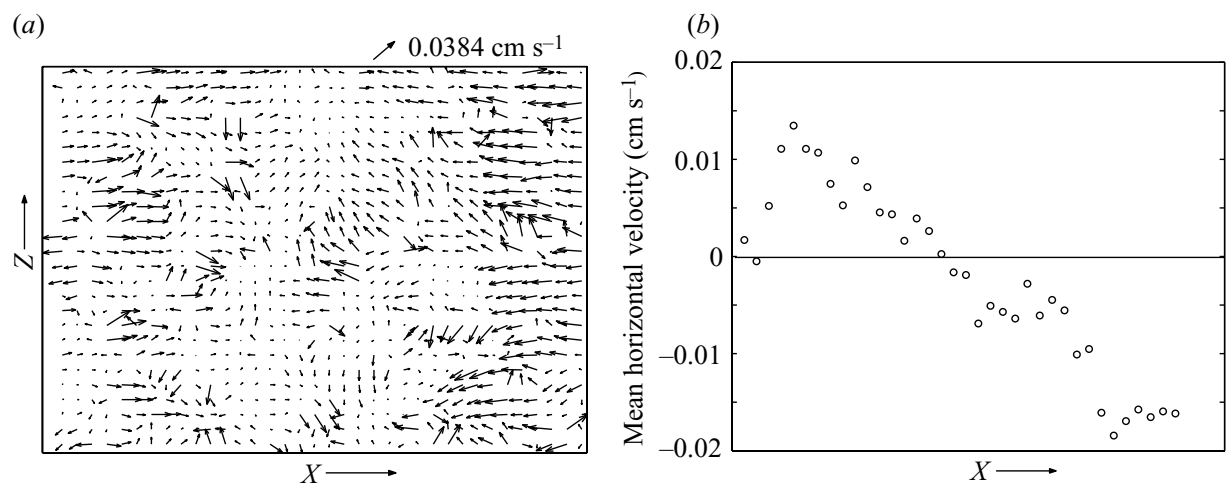

FIGURE 6. The near-wall velocity of sheet plumes at $R a_{w}=6.39 \times 10^{10}$ in a zoomed portion of figure $5(a)$. The image size is $10.8 \mathrm{~cm} \times 8.1 \mathrm{~cm}$. (a) The near-wall velocity of movement of the lighter fluid estimated by the spatial intensity correlation method of Gendrich \& Koochesfahani (1996). (b) Variation of $U$ velocity averaged in the $Z$-direction with $X$.

Figure $6(a)$ shows the velocities estimated using the spatial intensity correlation technique of Gendrich \& Koochesfahani (1996) from a zoomed portion $(10.8 \mathrm{~cm} \times$ $8.1 \mathrm{~cm})$ of the near-wall image of figure $5(a)$. The correlation was calculated between two images $5 \mathrm{~s}$ apart and then averaged over a set of 6 such pairs $0.2 \mathrm{~s}$ apart. The nearwall velocity map of the movement of the lighter fluid clearly shows the presence of a mean shear in the horizontal direction near the left- and right-hand walls. Figure $6(b)$ shows the variation of $Z$-averaged $U$ velocity with $X$, calculated from figure 6(a). The mean shear velocity varies almost linearly along the $X$-direction. The velocity in figure $6(b)$ represents the average velocity at the laser sheet location owing to the mean wind and will be within the velocity boundary layer. Note that the maximum near-wall velocity is an order smaller than the plume column rise velocities discussed in $\S 4.1 .1$; the Reynolds number based on the maximum near-wall velocity and $Z_{w}$ is 0.03 .

\subsubsection{Near-wall dynamics}

The plume sheets are constantly moving, mostly owing to the interactions among themselves. Two main dynamics are observed near the wall:

(a) A new plume is initiated at a point, usually in a vacant region, and then becomes elongated to a sheet.

(b) Adjacent plumes which are parallel and close to each other merge. The merger is due to the entrainment field between the plumes.

As we have seen, the effect of the wind or large-scale circulation is to align the sheet plumes in the direction of the shear (right- and left-hand sides of figure $5 a$ ). In regions of shear (created by the mean wind) the elongation of the initiation point is in the direction of shear. The main sheet plume segments also become elongated to touch each other.

In the absence of a wind, as at lower $R a$, the plumes are randomly aligned. Figure 7 shows the dynamics in a region where the mean shear is small. The images are taken from the centre region of planforms, below the rising plume column, similar to that in figure $5(a)$, i.e. the shear predominant areas, having a horizontal mean shear are on the right- and left-hand side of each image in the figure. The plumes in the low mean shear areas are initiated as points in vacant areas (point $\mathrm{A}$ in figure $7 a$ ) and becomes elongated to sheets. See the evolution of the plume sheet initiated at point A in figures $7(a), 7(b)$ and $7(c)$. The direction of elongation is probably decided by 
(a)

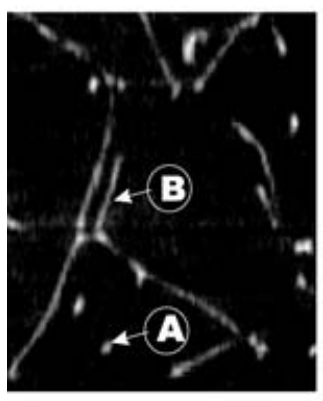

(b)

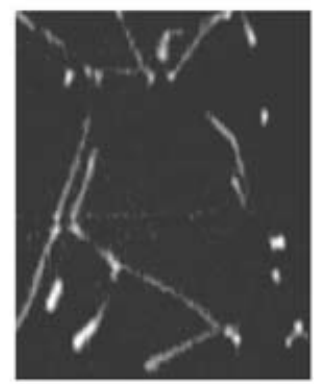

(c)

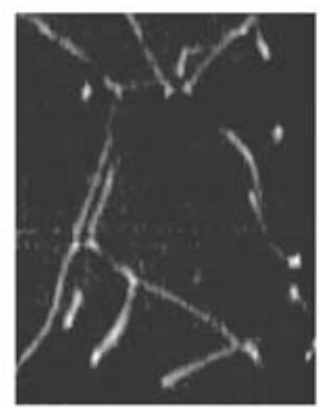

(d)

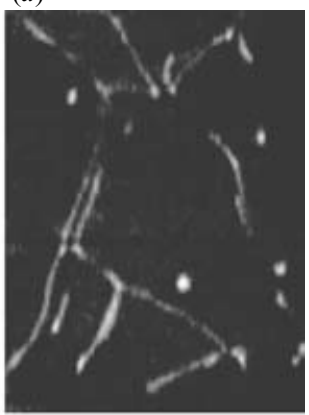

(e)

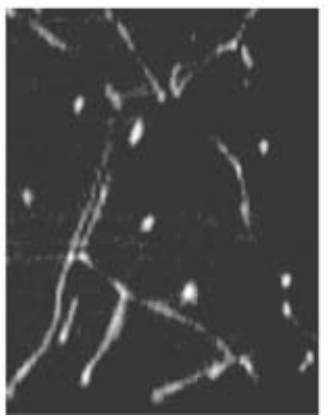

(f)

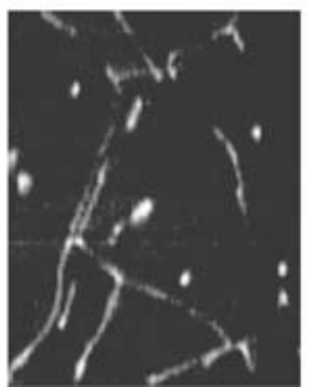

FiguRE 7. Near-wall plume dynamics in a weak mean shear region from the centre of a structure similar to that in figure 5(a). The images are $2 \mathrm{~s}$ apart, time increasing from $(a)$ to $(f)$ at the same $R a_{w}$ as in figure 6 . At point (A) in $(a)$ a plume initiates as a point and becomes elongated with time. At point (B), two locally parallel plumes merge.

a combination of the nature of the boundary-layer instability, the entrainment flow of the nearby plumes and the effect of the rising plume column. The elongation is at a slower rate than in the shear predominant areas, the reason why more points (vis-à-vis the shear predominant areas) are seen in the shear-free areas. There are also locally parallel plumes, close to each other, which merge ( see point B in figure $7 a$ ). The plumes merge in a few seconds, a much smaller time scale than the 4 min time scale of the large-scale circulation.

The planform structure of figure $5(a)$ is observed consistently for about $5 \mathrm{~h}$ from the time of figure $5(d)$, i.e. until $R a_{w} \sim 5.1 \times 10^{10}$.

\section{2. $R a_{w} \sim 10^{11}$}

Convection at a higher Rayleigh number (earlier in the experiment) of $2.04 \times 10^{11}$ in the same set-up, $(A R=0.65)$ shows the planform plume structure of figure $8(a)$. Figure $8(c)$ shows the side view in a vertical plane $2 \mathrm{~cm}$ from a sidewall of the test section (i.e. $2 \mathrm{~cm}$ from the bottom of figure $8 a$ ).

A larger number of sheet plumes are seen in figure $8(a)$ than in figure $5(a)$. The planform displays circular patches of aligned lines originating from a lower plume density area in the centre of these patches. The vertical view of figure $8(c)$ shows that small near-wall plumes merge into rising plume columns of larger size. The rising of these plume columns creates a downward flow which impinges on the wall, creating the circular, relatively plume-free patches seen in figure $8(a)$. The aligned lines around the patch in figure $8(a)$ are created owing to the near-wall mean wind, directed radially 

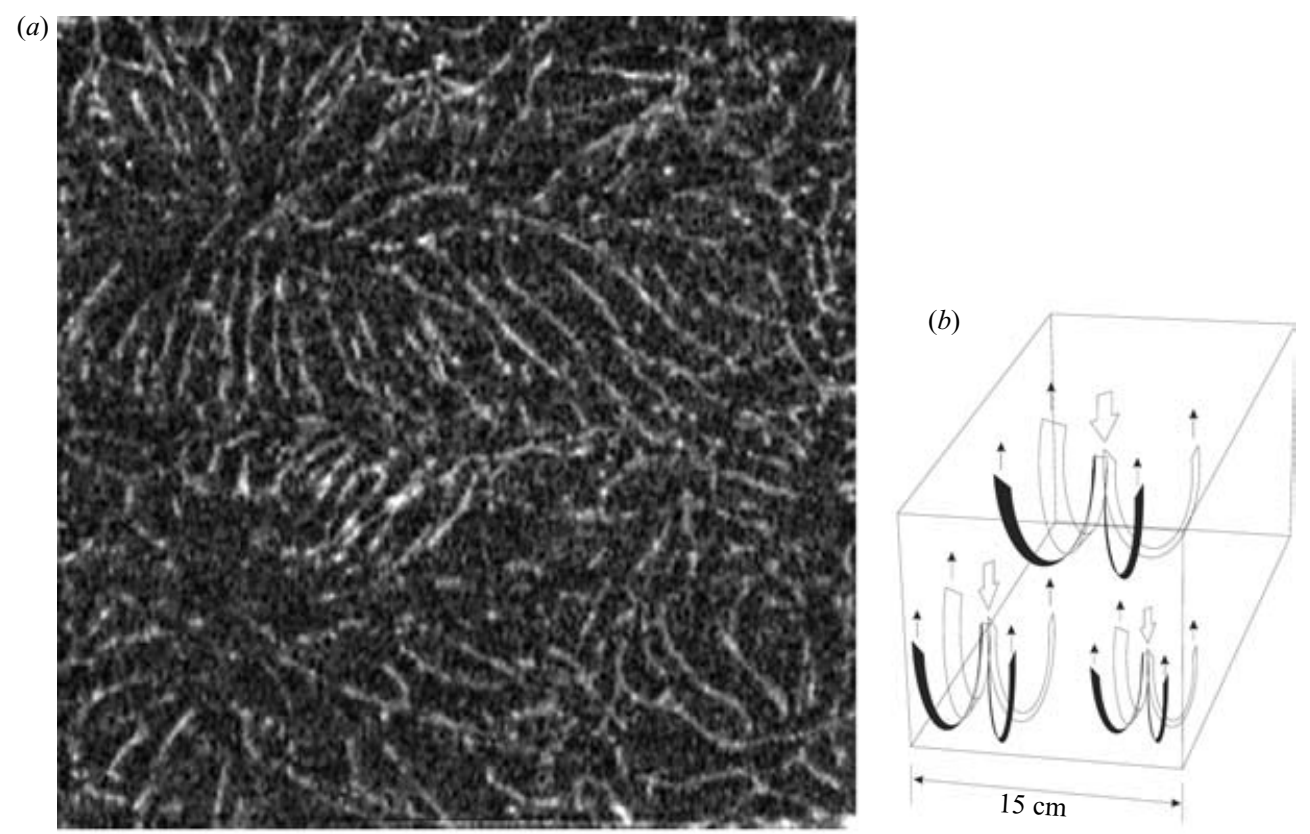

(c)

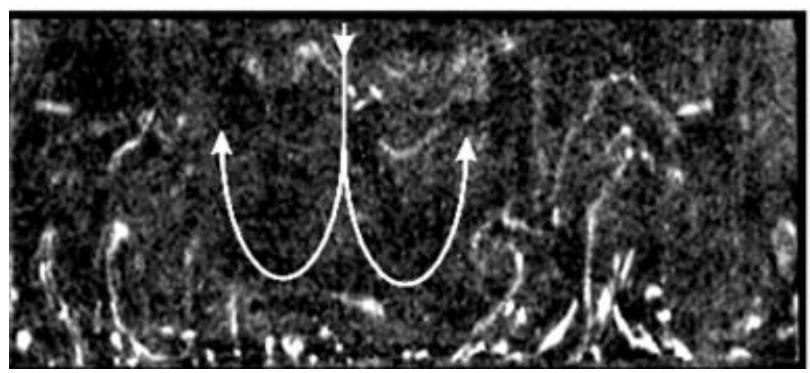

FIGURE 8. Multiple large-scale flow cells at $A R=0.65$ and $C_{T}^{o}=10 \mathrm{gl}^{-1}$ (Expt 10jan). (a) Planform of plume structure at $R a_{w}=2.04 \times 10^{11}, \Delta C_{w}=3.205 \mathrm{~g}^{-1}$. Image size is $15 \mathrm{~cm} \times 15 \mathrm{~cm}$. See table 2 for details of parameters. $(b)$ Schematic of the multiple large-scale flow cells. (c) Side view of plume structure in a vertical plane $2 \mathrm{~cm}$ from the bottom of figure $8(a)$ at $R a_{w}=2.03 \times 10^{11}$, image size is $15 \mathrm{~cm} \times 6.7 \mathrm{~cm}$.

outward from the plume-free patch. This mean wind also sweeps the smaller near-wall plumes into the larger plume columns, as seen in the smaller inclined near-wall plumes in figure $8(c)$. Figure $8(a)$ shows two large-scale flow cells on the left-hand side of the image and one small cell at the bottom right-hand side; a schematic of this flow is shown in figure $8(b)$. The plume column rise velocity is about $0.3 \mathrm{~cm} \mathrm{~s}^{-1}(\operatorname{Re}=772.7)$ while the downward velocities are larger at $\sim 0.8 \mathrm{~cm} \mathrm{~s}^{-1}(R e=2060)$. The correlation of Lam et al. (2002) for the present parameters gives $R e=670.3\left(V_{L S}=0.26 \mathrm{~cm} \mathrm{~s}^{-1}\right)$. The free-fall velocity in the present case when $\Delta C_{w}$ acts over the layer height $H$ is $V_{f f}=7.14 \mathrm{~cm} \mathrm{~s}^{-1}$, an overestimate by an order of magnitude from the plume column rise velocities. The Deardorff velocity for figure $8(c)$ is $0.31 \mathrm{~cm} \mathrm{~s}^{-1}$, of the same order as the plume column rise velocity. The maximum near-wall mean velocity is around $0.06 \mathrm{~cm} \mathrm{~s}^{-1}$, larger than the velocity obtained in figure 6(a) owing to the increase in the Rayleigh number; the near-wall mean shear increases with increase in $R a_{w}$. This increase seems to have two components, namely, the increase in plume column rise 
(a)

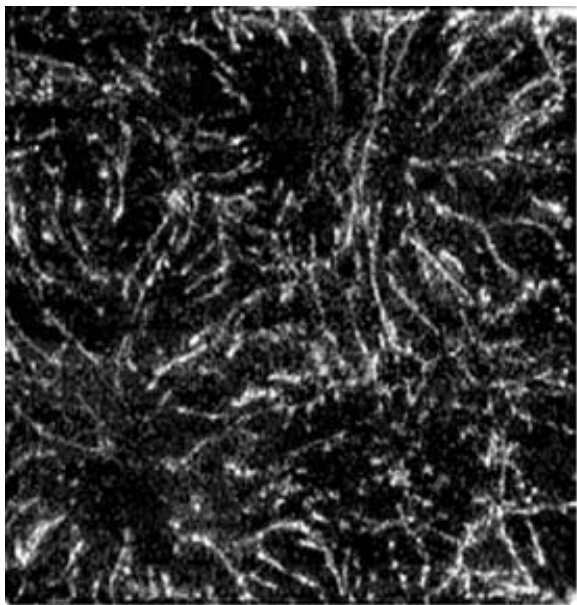

(b)

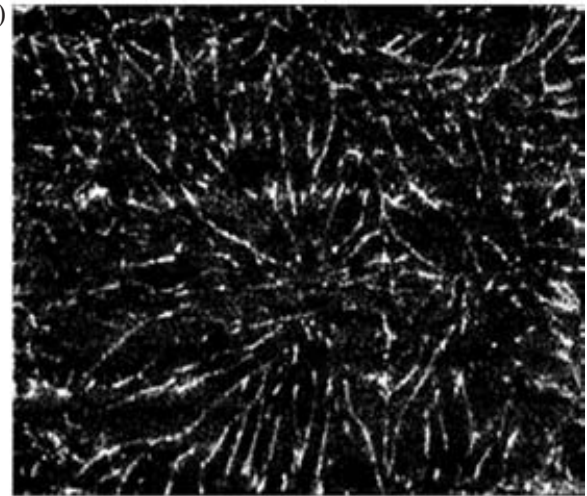

(c)
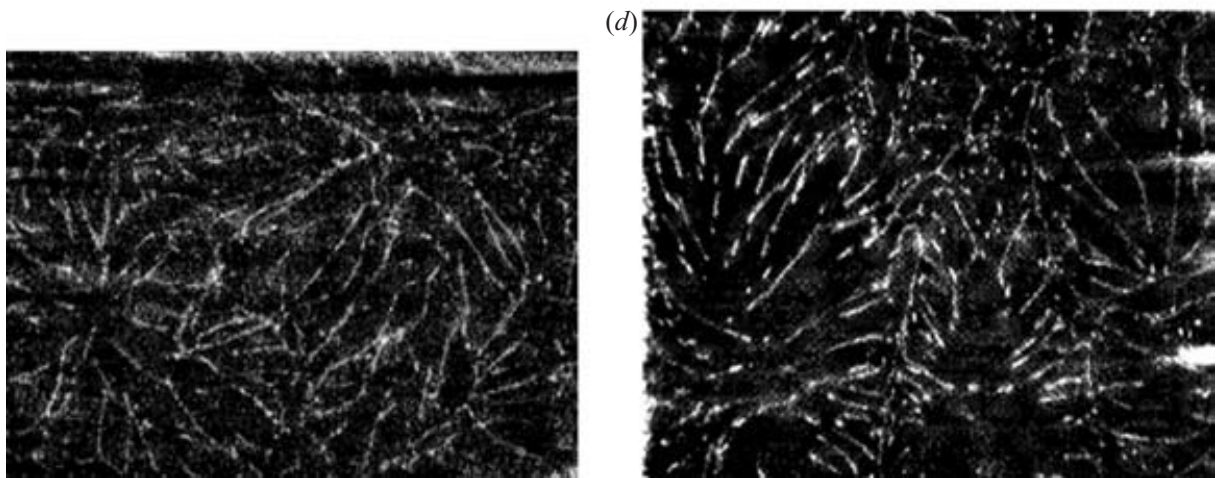

FIGURE 9. Planform structure with decreasing $R a_{w}$ for $A R=0.65$ for experiments with $C_{T}^{o}=10 \mathrm{~g} \mathrm{l}^{-1}$. Parameter details are shown in table 2. (a) Exp. 10jan, 22.5 min prior to figure 8( $\left.a\right)$, $R a_{w}=2.07 \times 10^{11}$. (b) Exp. 10jan, $R a_{w}=1.87 \times 10^{11}$. (c) Exp. 10jan, $R a_{w}=1.69 \times 10^{11}$. $(d)$ Exp. 4feb, $R a_{w}=1.67 \times 10^{11}$.

velocities due to the larger driving potentials and the increase due to the reduction in area available for the downflow due to the presence of multiple rising plume columns. The Deardorff velocity correctly estimates the average large-scale flow velocity at the higher Rayleigh number also.

The near-wall dynamics was similar to that described in $\S 4.1 .2$, but with a shorter time scale. The main differences in the planforms at $R a_{w} \sim 10^{11}$ from that at $R a_{w} \sim 10^{10}$ are in the large-scale circulation cell nature (multiple toroidal cells as against twocounter-rotating cylindrical cells) and the larger number of plumes at the higher $R a_{w}$.

\subsubsection{Changing large-scale flow patterns}

At the higher Rayleigh numbers, the large-scale flow cell patterns are seen to change with time. The various planform images in order of decreasing $R a_{w}$ are shown in figure $9(a), 8(a), 9(b), 9(c), 9(d)$ and $5(a)$. Table 2 shows the relevant parameters for these images. The entries are arranged as per decreasing $R a_{w}$ (or flux Rayleigh number, $R a_{q}$ ).

Figure $9(a)$ shows the planform plume structure 22.5 min prior to the image shown in figure $8(a)$. The circulation cell at the top right-hand side in figure $9(a)$ has shifted 
to the top left-hand side in figure $8(a)$. The characteristic time of one large-scale flow circulation spanning the cell volume $\left(H / W_{*} \sim 1 \mathrm{~min}\right)$ and the time scale associated with the near wall merging of sheet plumes ( $\sim$ few seconds) are much smaller than the time scale for the shifting of large-scale flow cells. The time scale for the bulk concentration change $\Delta C /\left(\mathrm{d} C_{T} / \mathrm{d} t\right)$ is much larger $(\sim 600 \mathrm{~min})$; (quasi)statistical stationary nature can hence be assumed. Observations of changing large-scale flow direction at similar $R a$ have also been made by Niemela et al. (2001).

Figure $9(b)$ shows the planform after about one and a half hours after the start of the experiment. The image shows the signature of a large-scale circulation impinging at the centre of the cross-section area. The planform shifts between multiple cells and single cells (formed due to merging of multiple cells) with decreasing $R a_{w}$. One important feature that is observed is the reduction in the number of plume sheets or the increase in the average spacing between adjacent plumes with reduction in Rayleigh number. For example, compare figures $9(a), 9(d)$ and $5(a)$. At around the same $R a_{w}$ as in figure $9(c)$, in another experiment, we notice the beginning of the breaking up of the circular cells to give arrays of aligned plumes as shown in figure $9(d)$. This might be the possible route to the plan forms obtained at the beginning of low concentration $\left(C_{T}^{o}=3 \mathrm{~g}^{-1}\right)$ experiments shown in figure $5(a)$.

\section{Plume structure at $A R=0.435$}

The planform plume structure with $H=23 \mathrm{~cm}$ and the test-section cross-section of $10 \mathrm{~cm} \times 10 \mathrm{~cm}$ (i.e. $A R=0.435$ ) at $R a_{w}=2.03 \times 10^{11}$ is shown in figure $10(a)$. The image shows the full test-section cross-section. The presence of aligned plumes on the right-hand side of the figure and the progressive decrease of alignment from right to the left indicates a single clockwise large-scale flow cell spanning the full test-section cross-section, shown schematically in figure $10(b)$. It is inferred that the large-scale flow spans the whole height of the test section as motion at the top surface of the liquid is observed to be in the opposite direction to the mean shear near the membrane surface. Figure $10(c)$ shows the plume structure in a vertical plane. Plumes combine and rise along the left-hand wall, feeding the mean circulation, which in turn sweeps the near-wall plumes from the right to the left. The major difference from the structure for $A R=0.65$ at similar Rayleigh numbers, shown in figure $8(a)$, is the absence of multiple large-scale flow cells.

In addition to a single cell parallel to the walls (figure 10a), a variety of planforms and large-scale flow patterns were observed. The large-scale circulation in figure 11(a) is rotating clockwise, impinging on the top right-hand corner and creating a mean shear near the membrane along the diagonal from top right to bottom left. In most cases, it can be seen that a relatively plume-free area is created when the large-scale flow is aligned along the diagonal (see figure 11a). The two adjacent sidewalls focus the overall circulation into a downward moving column of fluid in the corner, which creates the plume-free area with near-wall mean shear direction away from this patch. At lower driving potentials, in some experiments, the plumes were seen to combine at the centre, resulting in a column of rising fluid at the centre of the cross-section, creating a toroidal structure of the large-scale flow, as seen in figure 11(b). Table 3 shows the relevant parameters corresponding to each image. At further low $R a_{w}$, the alignment becomes quite weak, the plume sheets are randomly oriented, the lateral movement of plumes due to the entrainment effect of nearby plumes becomes larger, and the planform structure looks similar to that observed by Theerthan \& Arakeri (2000). 

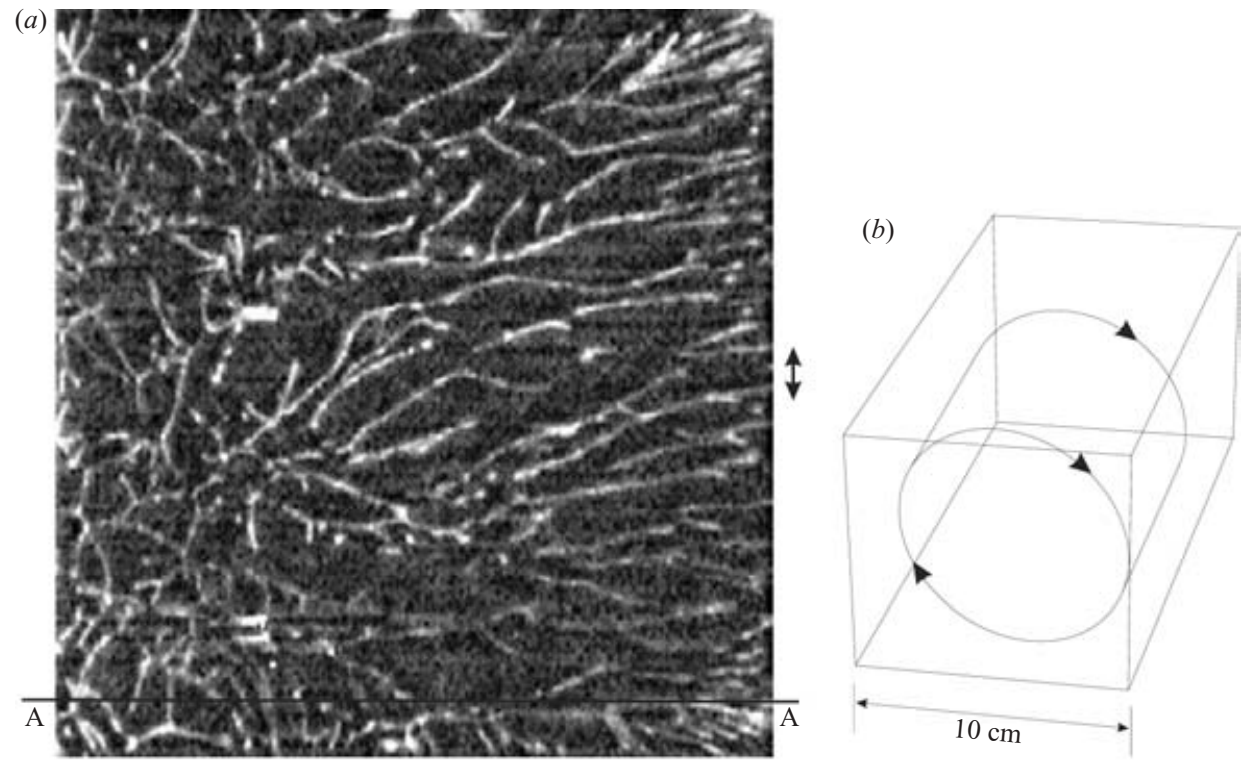

(c)

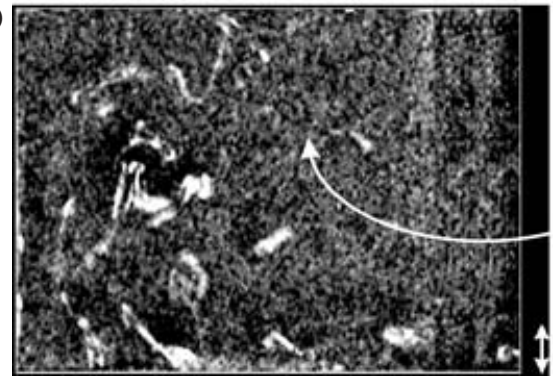

Figure 10. Single large-scale flow cell at $A R=0.435$ and $C_{T}^{o}=10 \mathrm{~g} 1^{-1}$ (Exp 18dec). (a) Planform of plume structure at $R a_{w}=2.0340 \times 10^{11}, \Delta \rho / \rho_{w}=2.3 \times 10^{-3}$, image size is $10 \mathrm{~cm} \times$ $10 \mathrm{~cm}$. (b) Schematic of the large-scale flow cell. (c) Vertical plume structure at $R a_{w}=$ $2.05 \times 10^{11}$, image size is $7.25 \mathrm{~cm} \times 4.67 \mathrm{~cm}$.

$\begin{array}{lcccccccc}\text { Exp. } & \text { Figure } & \Delta C_{w} & R a_{w} \times 10^{11} & \begin{array}{c}q \\ \left(\mathrm{mg} \mathrm{cm}^{-2} \mathrm{~min}^{-1}\right)\end{array} & R a_{q} \times 10^{14} & \begin{array}{c}W_{*} \\ \left(\mathrm{~cm} \mathrm{~s}^{-1}\right)\end{array} & \begin{array}{c}\text { Time } \\ (\mathrm{min})\end{array} & \text { Orientation } \\ 18 \mathrm{dec} & 10(a) & 3.2 & 2.03 & 0.1094 & 1.8 & 0.31 & 20.17 & \text { Parallel to wall } \\ 20 \mathrm{nov} & 11(a) & 2.83 & 1.8 & 0.079 & 1.3 & 0.276 & 209.83 & \text { Diagonal } \\ 18 \mathrm{dec} & 11(b) & 2.62 & 1.67 & 0.08 & 1.32 & 0.2773 & 263.17 & \begin{array}{c}\text { Rising column } \\ \text { at centre }\end{array}\end{array}$

TABLE 3. Parameters corresponding to planform structures for $A R=0.435$.

The process of shifting the large-scale flow direction is very slow compared to the time period of one circulation of the mean wind. Table 3 shows the time of the image from the start of the experiment. The time period between the clear change in structures in the experiment of $18 \mathrm{dec}$ was about an hour. There are transition planform structures in between the images shown in figures 10(a) to 11(b) which are 

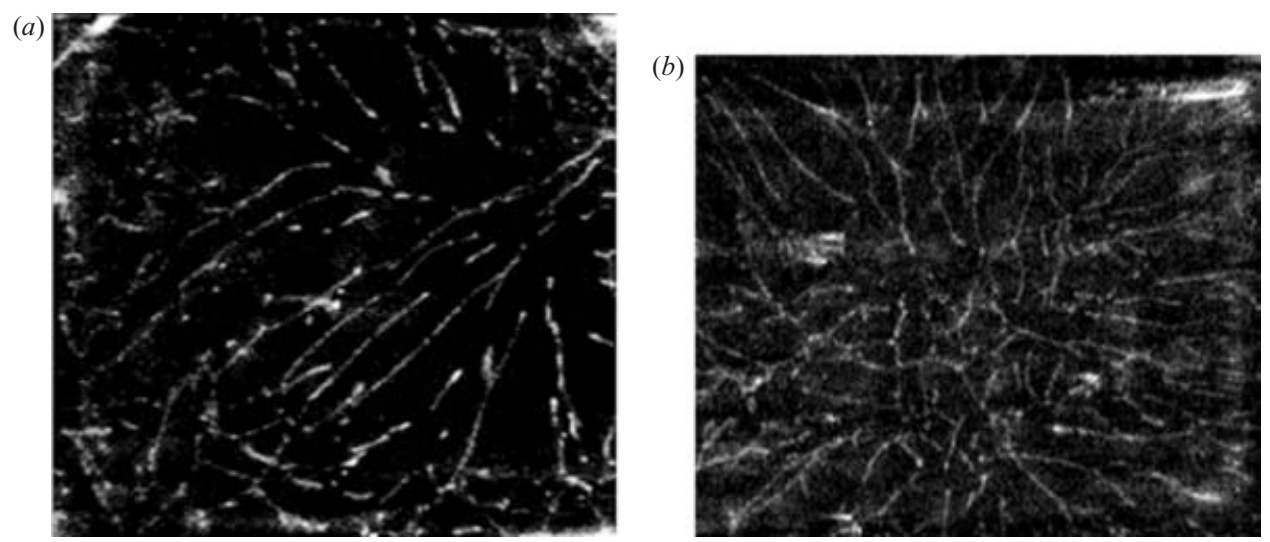

FIGURE 11. Planform structure with decreasing $R a_{w}$ for $A R=0.435$. Parameters details are shown in table 3 . In figure $11(a)$ the mean wind is along the diagonal starting from the top right-hand corner. In figure $11(b)$ the large-scale flow is toroidal, with a column rising in the centre. (a) Exp. 20nov, $R a_{w}=1.802 \times 10^{11}$. (b) Exp. $18 \mathrm{dec}, R a_{w}=1.665 \times 10^{11}$.

a combination of the above clear regimes. These are expected to occur when the large-scale flow is switching from one type to another.

\section{Plume spacing distribution}

The sheet plumes are the result of the gravitational instability of the boundary layers in between them; the measurement of the spacings of the sheet plumes helps us to form inferences about the nature of these boundary layers in $\S 7$. The plume spacings are calculated from the coordinates of adjacent plume sheets input manually by mouse clicks. The coordinates are selected judiciously so that the spacings are measured normal to the plume sheets wherever possible. The measurements are made all over the image to cover the whole range of spacings in the image. As the plume sheets are not always parallel, the location of measurement would affect the calculated spacings. However, with a large enough sample, the mean spacing and the distribution of spacings would be representative of the actual spacing distribution in the planform image. The average number of samples in each image varied depending on the number of plume sheets in the image. At the highest $R a_{w}$, for planforms similar to that in figure 8 , the average sample size is about 500 . The number of plumes, and thus the sample size, reduced with decrease in $R a_{w}$. The sample size at the lowest $R a_{w}$ is about 225. The statistics are improved by taking the spacings measured from about three images a short time apart so that the images are almost at the same $R a_{w}$. The outliers in the data are removed using the criteria suggested by Frank \& Althoen (2002) for skewed distributions.

\subsection{Mean spacing variation}

Figure 12(a) shows the variation of mean plume spacing, $\bar{\lambda}$ with $R a_{w}$. The plot shows the spacing measurements from the low and the high concentration experiments in the two aspect ratio tanks. The solid line is a theoretical prediction explained in $\S 7.1$. The mean plume spacing clearly reduces with increase in $R a_{w}$. No clear dependence on $A R$ can be found. The plot also shows that there is about $25 \%$ variation in the spacings at the same $R a_{w}$. This variation is expected to be due to the human error 

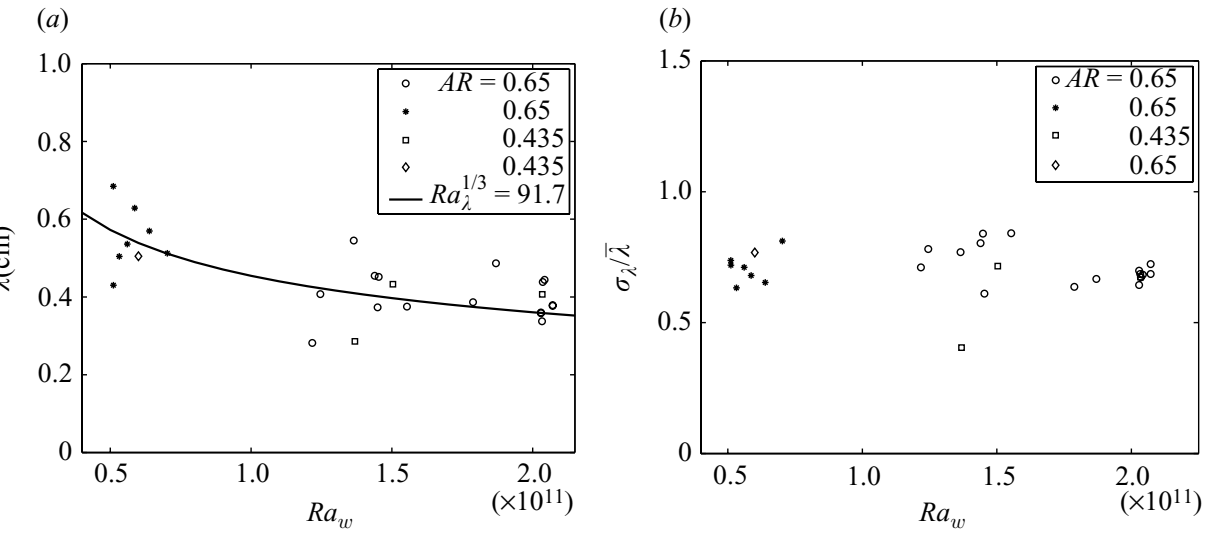

FIGURE 12. Variation of the mean plume spacings and the normalized standard deviation of the plume spacings with the Rayleigh number. The line in $(a)$ is a prediction from the model discussed in $\S 7.1$. (a) Mean plume spacing $\bar{\lambda}$. (b) Normalized standard deviation of plume spacings, $\sigma_{\lambda} / \bar{\lambda}$.

(a)

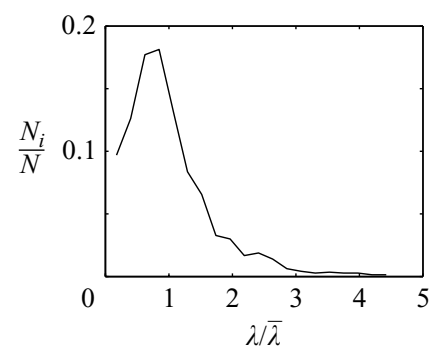

(b)

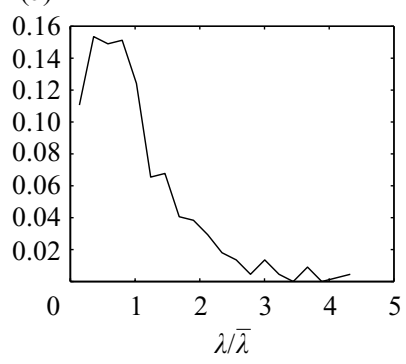

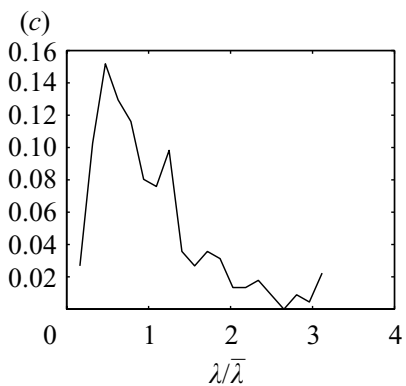

FiguRE 13. Histogram of plume spacings of different planforms at decreasing $R a_{w}$. (a) Planform as in figure $8,(b)$ as in figure $9(c),(c)$ as in figure $5(a) .(a) \tilde{\lambda}=0.3508 \mathrm{~cm}$; sample size $=1434 ; \sigma_{\lambda}=0.2375 ;-, R a_{w}=2.03 \times 10^{11}$. (b) $0.4241 \mathrm{~cm} ; 443 ; 0.3214 ; 1.44-1.46 \times 10^{11}$. (c) $0.5695 \mathrm{~cm} ; 224 ; 0.3722 ; 5.9 \times 10^{10}$.

in measurements, insufficient sample size and differences in location of measurement between the images.

Figure $12(b)$ shows the dependence of the normalized standard deviation of the plume spacings $\sigma_{\lambda} / \bar{\lambda}$, on $R a_{w} . \sigma_{\lambda} / \bar{\lambda}$ is about 0.7 ; the variance increases proportionally to the mean plume spacing. The increase in $\sigma_{\lambda}$ with decrease in $R a_{w}$ shows that the plume spacings become spread out over a wider range at lower Rayleigh numbers. Thus an increase in Rayleigh number (and hence increase in flux) results in plumes which are closely spaced (lower mean spacing) and with less variation in the spacing between plumes (less $\sigma_{\lambda}$ ). The number of plumes per unit area increases with $R a_{w}$. Note that this observation is not in agreement with the modification suggested by Grossman \& Lohse (2004) to their earlier theory.

\subsection{Spacing statistics}

The histograms of the plume spacings (normalized with the mean) at three $R a_{w}$ are shown in figure 13. The $R a_{w}$ values cover the range shown in figure $12(a)$. Figure $13(a)$ is obtained from the spacing measurements of three images $15 \mathrm{~s}$ apart 

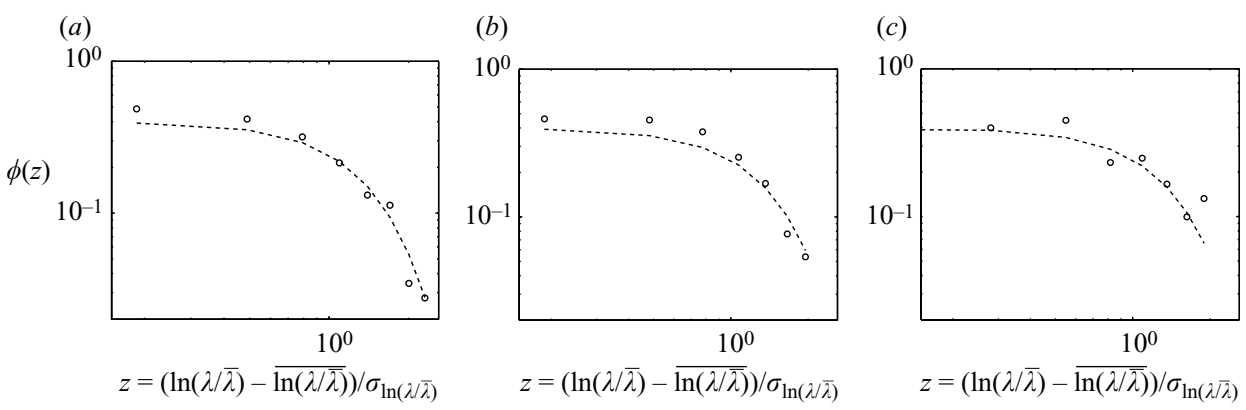

Figure 14. Probability density function of logarithm of plume spacings in the standardized form along with standard normal curve at decreasing $R a_{w}$. Data from the corresponding figures $13(a)$ to $13(c),---$, standardized normal curve. $(a) \overline{\ln (\lambda / \lambda)}=-0.2239 ; \sigma_{\ln (\lambda / \bar{\lambda})}=0.7058$; $\mathrm{O}, R a_{w}=2.03 \times 10^{11} .(b)-3059 ; 0.8549 ; 1.44-1.46 \times 10^{11} .(c)-0.3722 ; 0.6746 ; 5.9 \times 10^{10}$.

so that the $R a_{w}$ change is negligible. The planform for these statistics corresponds to figure 8 . The spacing measurements from 3 images about 2 min apart are used for calculating figure $13(b)$. The planform corresponds approximately to that shown in figure $9(c)$. Figure $13(c)$ is from a single image of the low-concentration experiment, which exhibits planform structure similar to that in figure 5(a). The spiky nature of figure $13(c)$ is due to the low sampling size.

Figure 13 shows that even though the mean and the variance of plume spacings increase with decreasing $R a_{w}$, all the histograms exhibit the same form of a skewed distribution with a long tail. This common form of the histograms is seen more clearly when the logarithm of the normalized plume spacings is plotted in the standard form. Figure 14 shows $(\ln (\lambda / \bar{\lambda})-\overline{\ln (\lambda / \bar{\lambda})}) / \sigma_{\ln (\lambda / \bar{\lambda})}$ plotted as a probability density function; here, $\overline{\ln (\lambda / \bar{\lambda})}=$ mean of $\ln (\lambda / \bar{\lambda})$ and $\sigma$ indicates variance. The standard normal probability density function, $(1 / \sqrt{2 \pi}) \exp \left(-\frac{1}{2} \ln (\lambda / \bar{\lambda})^{2}\right)$, shown in the figures shows good agreement with the experimental measurements. Figure 14 hence shows that the probability distribution function of the plume spacings has a common lognormal form for the three visually different planform structures at the three different $R a_{w}$. This common form of the probability distribution function was seen for all the images analysed.

Based on this common form of the distribution function, we combine the standardized form of $\ln (\lambda / \bar{\lambda})$ at different $R a_{w}$ from all the images analysed in figure 12(a), and plot the probability distribution of 7355 spacings (figure 15). The standard normal probability distribution function fits the data quite well. Thus, the probability density function of plume spacings in turbulent-free convection in the range $4 \times 10^{10} \leqslant R a_{w} \leqslant 2 \times 10^{11}$ at $S c=602$ can be approximated by the standard log-normal curve. The parameters that describe the standard log-normal curve are the mean $\ln (\lambda / \bar{\lambda})$, and the standard deviation $\sigma_{\ln (\lambda / \bar{\lambda})}$. The variation of these parameters for the present experimental range is shown in figure 16 . The plume spacing distribution in the present experiments is completely described by the plots in figures 15, 16(a) and $16(b)$. We also found that all the plume structures have a common underlying multifractal spectrum of singularities, which was proposed to be due to the common generating mechanism of these structures (Puthenveettil \& Arakeri 2005). The connections of the common log-normal spacing distribution to the common multifractal nature of the plume structure should be investigated. 


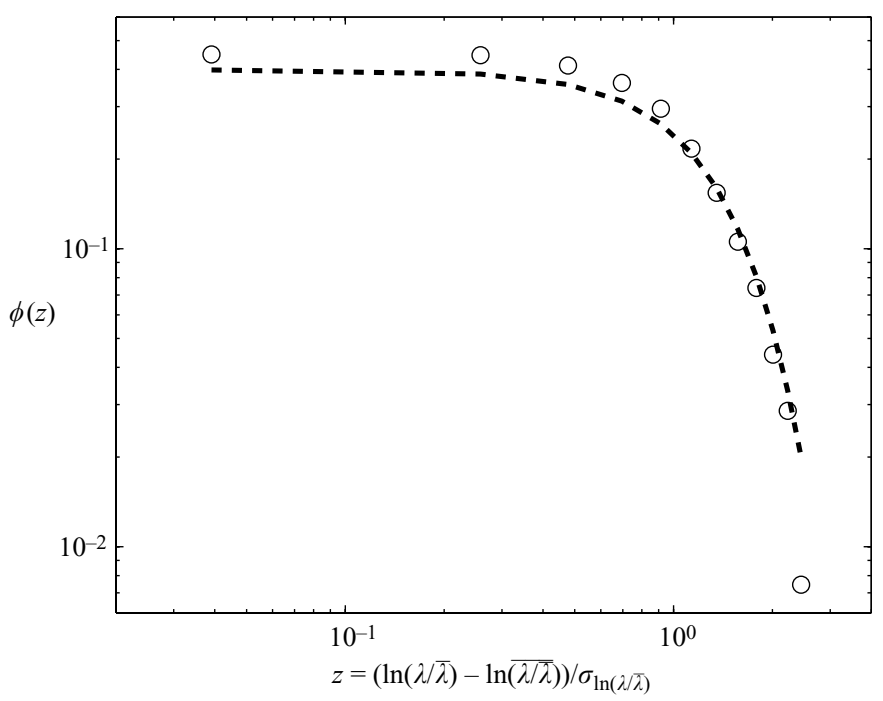

FIGURE 15 . The probability distribution function of plume spacings in turbulent free convection for $4 \times 10^{10} \leqslant R a_{w} \leqslant 2 \times 10^{11}$ at $S c=602$. O, measurements; - - -, standardized normal.

(a)

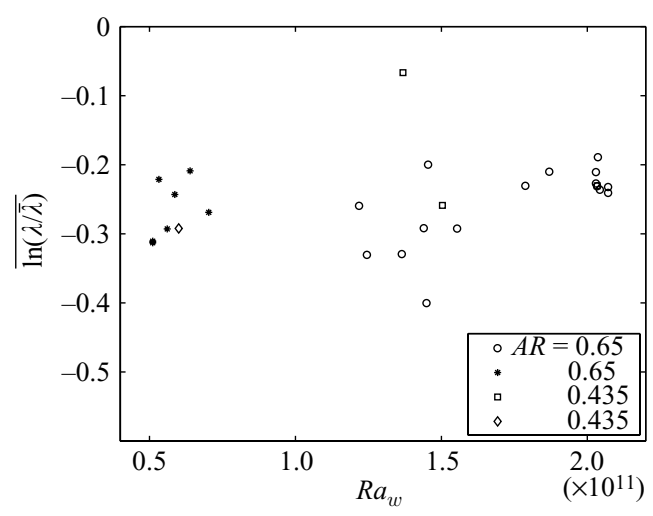

(b)

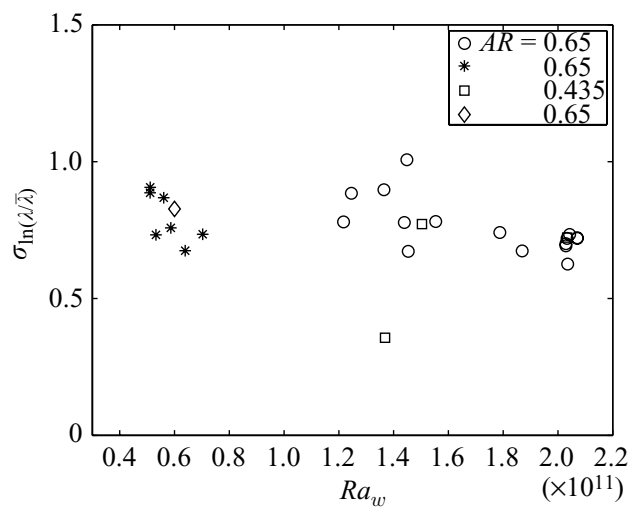

FiguRE 16. Variation of the standardizing variables, $\overline{\ln (\lambda / \bar{\lambda})}$ and $\sigma_{\ln (\lambda / \bar{\lambda})}$, in figure 15 with Rayleigh number. (a) Mean of logarithm of normalized plume spacings. (b) Standard deviation of logarithm of normalized plume spacings.

\section{The hypothesis of laminar natural convection boundary layers and its verification by a near-wall model}

The literature review in $\S \S 1.2,1.3$ and 1.4 showed that there is considerable controversy about the nature of boundary layers in high-Rayleigh-number turbulent free convection. The planforms of the near-wall plume structure from the present study show that the dominant near-wall coherent structures in high $\mathrm{Ra}$, high $\mathrm{Sc}$ turbulent convection are sheet plumes. These plumes are log normally distributed about a mean spacing and move about laterally on the horizontal surface. Plumes are the result of gravitational instability of the boundary layers between them. We hypothesize that the boundary layers between the sheet plumes are laminar natural convection boundary layers. These boundary layers grow perpendicular to the plume 
(a)

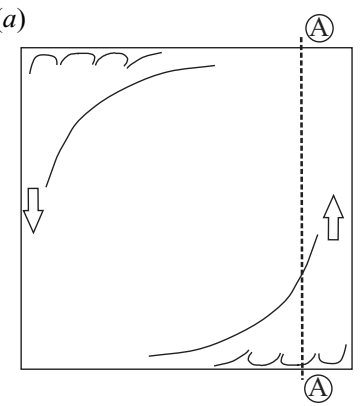

(b)

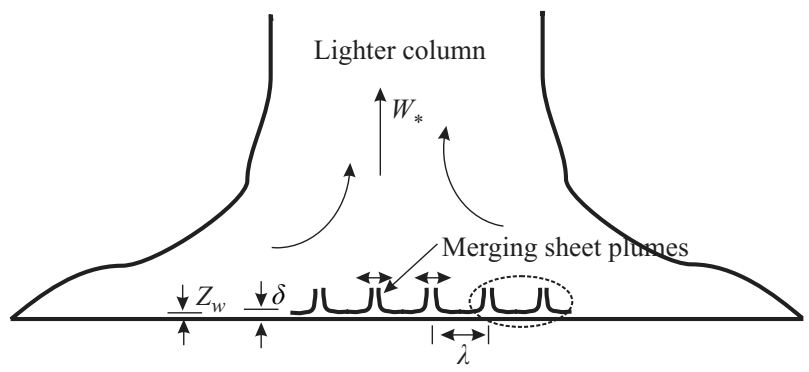

(c)

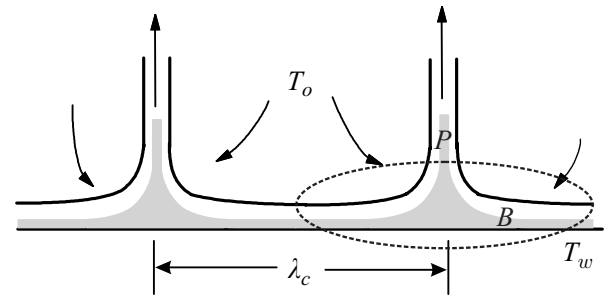

(d)

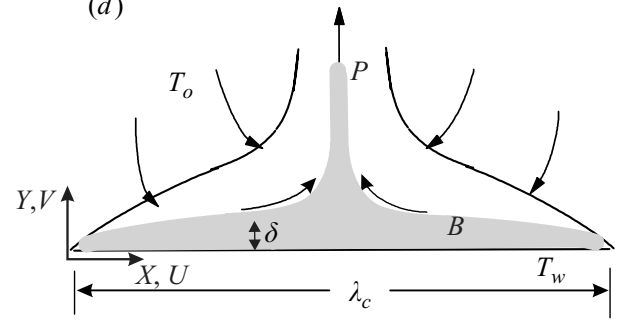

FIGURE 17. Schematic of the large-scale flow and the near-wall model. B indicates boundary layer and $\mathrm{P}$ indicates plume. The lines show the edge of the velocity boundary layer and the shaded regions show the thermal boundary layer and plume. The dashed ellipse in $(b)$ is shown as $(c)$ and the dashed ellipse in $(c)$ is shown as $(d) .(a)$ The overall large-scale flow schematic for a single circulation cell. (b) Side view of the vertical section in (A)-(A) plane showing near-wall laminar sheet plumes giving rise to column of lighter fluid, which drives the large-scale flow. (c) The model obtained as a regular array of the unit cell. $(d)$ The unit cell of the model.

sheets, driven by the horizontal pressure difference between the heavier ambient and the lighter boundary layer fluid. The predominant velocity in these boundary layers is horizontal. At some thickness of the boundary layer, when the Rayleigh number based on the boundary-layer thickness is of order 1000, gravitational instability sets in and the boundary layer turns upward to give rise to a sheet plume. These sheet plumes turn to mushroom-type structures at a very short height from the wall, and become organized into columns of lighter fluid, which drive the large-scale circulation (figure 17a,b). The large-scale flow in turn organizes the near-wall sheet plumes as well as the column of lighter fluid.

The laminar natural-convection boundary-layer hypothesis can be justified for two reasons. First, the boundary layers, before becoming unstable to give rise to plumes, could be expected to be laminar. Secondly, as we are dealing with near-wall flow where viscous effects are important, especially at large Prandtl numbers, the Reynolds numbers would be low. The Reynolds number based on the thickness of the fluid layer and the Deardorff outer velocity scale would be 800 . The Reynolds number based on near-wall scales, given by Townsend's velocity scales (equation (1.2)), and mean plume spacing $(\sim 0.6 \mathrm{~cm})$ is 0.8 .

We validate the hypothesis of laminar natural-convection boundary layers in the present experiments by comparing the prediction of the mean plume spacing from a model constructed from similarity solutions of laminar natural-convection boundary layers with the experimental measurements. The present model is an extension of the model of Theerthan \& Arakeri (1998) to high Prandtl number. 
7.1. A near-wall model for mean plume spacing at high Prandtl numbers from similarity solutions

We model the observed plume structure as a regular array of two-dimensional laminar natural convection sheet plumes that are spaced apart by a mean plume spacing $\lambda_{c}$. This regular array of 'unit cells' of two boundary layers giving rise to a sheet plume is expected to approximate the near-wall dynamics. The schematic of the unit cell is shown in figure $17(d)$, where B represents the boundary layer and $\mathrm{P}$ the plume. The model obtained as a regular array of this unit cell is shown in figure $17(c)$. The similarity solutions of Rotem \& Classen (1969) for a laminar natural-convection boundary layer over a horizontal surface for the asymptotic case of $\operatorname{Pr} \rightarrow \infty$ are used to approximate the near-wall boundary layers. We use temperature instead of concentration for the convenience of using Rotem \& Classen's (1969) notations; both are equivalent. From this model flow field, the expression for the mean values of any variable is calculated by averaging the similarity solutions over one unit cell. Equating the expression for the mean heat flux from the model to the correlation of Goldstein et al. (1990), an expression for the mean plume spacing is obtained.

The major assumptions used in this model are:

(a) Near-wall dynamics in turbulent convection can be approximated by that of laminar natural convection boundary layers.

(b) A two-dimensional model approximates the three-dimensional near-wall dynamics.

(c) The outer flow does not influence the flow near the wall.

The justification for the first assumption was described earlier. The second assumption can be justified because the near-wall flow is locally two-dimensional owing to the presence of sheet plumes. Besides, visualizations show that the neighbouring plumes are often nearly parallel. The outer flow affects the near-wall dynamics at very high $R a$. As noticed from visualizations, the first-order effect of the outer flow is to align the sheet plumes in the direction of the mean wind. The similarity solutions do not consider the effect of this external forcing on the boundary layers. However, as the effect of large-scale flow on the flux is weak, the predicted mean plume spacing - estimated by equating the flux - is expected to be fairly accurate. Further, at high Prandtl numbers the effect of large-scale flow is likely to be weak for the reasons discussed in $\S 1.5$.

\subsubsection{Similarity solutions for laminar natural-convection boundary layer as $\operatorname{Pr} \rightarrow \infty$}

For indirect laminar natural-convection boundary layers as $\operatorname{Pr} \rightarrow \infty$, Rotem \& Classen (1969, hereinafter referred to as RC) introduced

$$
\left.\begin{array}{l}
\tilde{y}=y R a_{c}^{1 / 5}, \tilde{u}=u G r_{c}^{-2 / 5} \operatorname{Pr}^{3 / 5}, \\
\tilde{v}=v G r_{c}^{-1 / 5} \operatorname{Pr}^{4 / 5}, \tilde{\pi}=\pi G r_{c}^{-4 / 5} \operatorname{Pr}^{1 / 5},
\end{array}\right\}
$$

as the asymptotically stretched inner variables that make the thickness of the thermal boundary layer unity in the stretched coordinates. The normalized variables in (7.1) are

$$
\left.\begin{array}{l}
y=\frac{Y}{L}, \quad x=\frac{X}{L}, u=\frac{U}{v / L}, v=\frac{V}{v / L}, \\
\pi=\frac{P-P_{\infty}}{\rho_{\infty} \nu^{2} / L^{2}}+\frac{g L^{3} Y}{v^{2}}, \theta=\frac{T-T_{\infty}}{T_{w}-T_{\infty}},
\end{array}\right\}
$$


where the uppercase letters denote dimensional variables, of the coordinate system shown in figure 17, and $L$ is the characteristic horizontal length. $L=\lambda_{c} / 2$ in our analysis, where $\lambda_{c} / 2$ is the critical length at which the boundary layer turns into a plume. $R a_{c}=g \beta\left(\lambda_{c} / 2\right)^{3} \Delta T_{w} / v \alpha$ is the Rayleigh number based on half the plume spacing and the corresponding Grashoff number, $G r_{c}=R a_{c} / P r$. Writing the continuity, Navier-Stokes and energy equation in terms of these inner variables and dropping terms of order $1 / P r$ and $1 / R a^{2 / 5}$, the thermal boundary-layer equations in the $P r \rightarrow \infty$ limit are obtained as (29) to (32) of RC. These equations are independent of the Prandtl number. The inertial terms drop out from the momentum equations. The horizontal momentum equation shows a balance of viscous resistance to the driving horizontal motion pressure gradient while the vertical momentum equation expresses a balance of the vertical pressure change and the buoyancy force.

The inner similarity variable for the thermal boundary layer,

$$
\tilde{\eta}_{1}=\tilde{y} x^{-2 / 5},
$$

is used to introduce similarity transformations. The similarity transformation for the streamfunction, motion pressure and temperature are

$$
\tilde{\psi}=x^{3 / 5} \tilde{F}_{1}\left(\tilde{\eta}_{1}\right), \quad \tilde{\pi}=x^{2 / 5} \tilde{G}_{1}\left(\tilde{\eta}_{1}\right), \theta=H_{1}\left(\tilde{\eta}_{1}\right) .
$$

In RC, the boundary-layer equations are now transformed into ordinary differential equations that are solved numerically for the velocity function $\tilde{F}_{1}$, the pressure function $\tilde{G}_{1}$ and the temperature function $\tilde{H}_{1}$. The solutions are given as functions of $\tilde{\eta}_{1}$ in figures 4 and 5 , and tabulated in tables $2 \mathrm{a}$ and $2 \mathrm{~b}$ in RC.

As $\mathrm{Pr}$ is not a variable in the boundary-layer equations, the solutions are accurate only to a factor of $\operatorname{Pr}^{a / 5}$ where $a$ is an integer. However, figures 4 and 5 of RC show that the plots of $\tilde{H}_{1}\left(\tilde{\eta}_{1}\right)$ and $\tilde{G}_{1}\left(\tilde{\eta}_{1}\right)$ at $\operatorname{Pr}=5$ to $\infty$ is not a strong function of $\operatorname{Pr}$. Hence, the $\operatorname{Pr} \rightarrow \infty$ solutions could be expected to hold for large Prandtl numbers.

\subsubsection{Averaging procedure}

Using the notation of RC, with the horizontal length scale $L$ chosen as $\lambda_{c} / 2$, the similarity variable can be written as

$$
\tilde{\eta}_{1}=\frac{Y}{\lambda_{c} / 2} R a_{c}^{1 / 5}\left(\frac{X}{\lambda_{c} / 2}\right)^{-2 / 5} .
$$

The non-dimensional plume spacing is given by

$$
\frac{\lambda_{c}}{Z_{w}}=R a_{\lambda_{c}}^{1 / 3}
$$

where $Z_{w}=\left(v \alpha / g \beta \Delta T_{w}\right)^{1 / 3}$ is the wall length scale in turbulent convection given by (1.3) and $R a_{\lambda_{c}}$, the Rayleigh number based on the critical plume spacing $\lambda_{c}$ is equal to $8 R a_{c}$.

The mean of any quantity is obtained by taking the spatial average over one unit cell. The mean, denoted by \langle\rangle , of any variable $\phi$ is

$$
\langle\phi\rangle=\frac{1}{\lambda_{c}} \int_{0}^{\lambda_{c}} \phi(X, Y) \mathrm{d} X=\frac{2}{\lambda_{c}} \int_{0}^{\lambda_{c} / 2} \phi(X, Y) \mathrm{d} X .
$$

As $\mathrm{d} X=\left(\lambda_{c} / 2\right) \mathrm{d} x$ from (7.2) and $\mathrm{d} x=-(5 / 2)\left(x^{7 / 5} / \tilde{y}\right) \mathrm{d} \tilde{\eta}_{1}$ from (7.3), a change of variable from $X$ to $\tilde{\eta}_{1}$ in (7.7) can be obtained using

$$
\mathrm{d} X=-\frac{5}{2} \frac{\lambda_{c}}{2} \tilde{y}^{5 / 2} \tilde{\eta}_{1}^{-7 / 2} \mathrm{~d} \tilde{\eta}_{1} .
$$


The limits of integration are changed to $\tilde{\eta}_{1}=\infty$ to $\tilde{\eta}_{1_{c}}$, where $\tilde{\eta}_{1_{c}}=\left.\tilde{\eta}_{1}\right|_{X=\lambda_{c} / 2}=\tilde{y}$, to obtain

$$
\langle\phi\rangle=\frac{5}{2} \tilde{y}^{5 / 2} \int_{\tilde{y}}^{\infty} \tilde{\eta}_{1}^{-7 / 2} \tilde{\phi}_{1}\left(\tilde{\eta}_{1}\right) \mathrm{d} \tilde{\eta}_{1} .
$$

As $\tilde{y}^{5 / 2}=2\left(Y / \lambda_{c}\right)^{5 / 2} R a_{\lambda_{c}}^{1 / 2}$ from (7.1), the mean value of any variable can finally be expressed in terms of the similarity variable $\tilde{\eta}_{1}$ as

$$
\langle\phi\rangle=5\left(\frac{Y}{\lambda_{c}}\right)^{5 / 2} R a_{\lambda_{c}}^{1 / 2} \int_{\tilde{\eta}_{1_{c}}}^{\infty} \tilde{\eta}_{1}^{-7 / 2} \tilde{\phi}_{1}\left(\tilde{\eta}_{1}\right) \mathrm{d} \tilde{\eta}_{1} .
$$

For example, substituting the temperature function $\theta=\tilde{H}_{1}\left(\tilde{\eta}_{1}\right)$ from $\mathrm{RC}$ for $\tilde{\phi}_{1}\left(\tilde{\eta}_{1}\right)$ in (7.10), the expression for the mean temperature distribution in the boundary layer is obtained as,

$$
\langle\theta\rangle=5\left(\frac{Y}{\lambda_{c}}\right)^{5 / 2} R a_{\lambda_{c}}^{1 / 2} \int_{\tilde{y}}^{\infty} \tilde{\eta}_{1}^{-7 / 2} \tilde{H}_{1}\left(\tilde{\eta}_{1}\right) \mathrm{d} \tilde{\eta}_{1} .
$$

Similar expressions for the velocities can also be obtained.

\subsubsection{Boundary-layer thickness}

The thermal boundary-layer thickness can be estimated from the similarity variable given by (7.5) as

$$
\frac{\delta}{\lambda_{c}}=\tilde{\eta}_{1_{\delta}} R a_{\lambda_{c}}^{-1 / 5}\left(\frac{X}{\lambda_{c}}\right)^{2 / 5}
$$

where, $\tilde{\eta}_{1_{\delta}}=3.5$, is the value of the similarity variable $\tilde{\eta}_{1}$ at the boundary-layer thickness. We have taken the boundary-layer thickness as the point at which $\theta=\tilde{H}_{1}\left(\tilde{\eta}_{1}\right)=$ 0.02 . The critical boundary-layer thickness $\delta_{c}$ is when $X=\lambda_{c} / 2$ in (7.12). The Rayleigh number based on $\delta_{c}, R a_{\delta_{c}}=g \beta \Delta T_{w} \delta_{c}^{3} / \nu \alpha$ can hence be expressed as,

$$
R a_{\delta_{c}}=2^{-6 / 5} \tilde{\eta}_{1_{\delta}}^{3} R a_{\lambda_{c}}^{2 / 5}
$$

\subsubsection{Heat flux}

The heat flux is given by

$$
Q=-\left.k_{f} \frac{\partial T}{\partial Y}\right|_{Y=0} .
$$

Combining $\mathrm{d} Y=\mathrm{d} y\left(\lambda_{c} / 2\right)$ from (7.2), $\mathrm{d} \tilde{y}=\mathrm{d} y R a_{c}^{1 / 5}$ from (7.1) and $\mathrm{d} \tilde{\eta}_{1}=\mathrm{d} \tilde{y} x^{-2 / 5}$ from (7.3), the relation for change of variables from $\mathrm{d} Y$ to $\mathrm{d} \tilde{\eta}_{1}$ in (7.14) is obtained as

$$
\mathrm{d} Y=\frac{1}{2} \lambda_{c} R a_{c}^{-1 / 5} x^{2 / 5} \mathrm{~d} \tilde{\eta}_{1}
$$

Transforming the variable from $Y$ to $\tilde{\eta}_{1}$ in (7.14), the local value of flux can be expressed as

$$
\tilde{Q}_{1}\left(\tilde{\eta}_{1}\right)=-k_{f} R a_{c}^{1 / 5} \frac{\tilde{\eta}_{1}}{\tilde{y}} \frac{\Delta T_{w}}{\lambda_{c} / 2} \tilde{H}_{1}^{\prime}(0) .
$$

Substituting $\tilde{Q}_{1}$ from (7.16) for $\tilde{\phi}_{1}$ in (7.10), and simplifying, the mean value of flux is obtained as

$$
\langle Q\rangle=-\frac{5 \times 2^{2 / 5}}{3} k_{f} \frac{\Delta T_{w}}{\lambda_{c}} R a_{\lambda_{c}}^{1 / 5} \tilde{H}_{1}^{\prime}(0) .
$$

Replacing $\lambda_{c}=R a_{\lambda_{c}}^{1 / 3}\left(v \alpha / g \beta \Delta T_{w}\right)^{1 / 3}$ in (7.17), the average heat flux scales as the ratio of the characteristic temperature $\Delta T_{w}$ over the characteristic wall length scale $Z_{w}$, 
and is given by

$$
\langle Q\rangle=-\frac{5 \times 2^{2 / 5}}{3} R a_{\lambda_{c}}^{-2 / 15} \tilde{H}_{1}^{\prime}(0) k_{f} \frac{\Delta T_{w}}{Z_{w}} \Rightarrow R a_{\delta}^{-1 / 3}=-\frac{5 \times 2^{2 / 5}}{3} R a_{\lambda_{c}}^{-2 / 15} \tilde{H}_{1}^{\prime}(0) .
$$

Note that this equation shows the $4 / 3$ power law observed in turbulent convection. The amount of heat $\left(\mathrm{W} \mathrm{m}^{-1}\right)$ that feeds the plume is $\langle Q\rangle \lambda_{c}$.

The Nusselt number, $N u=\langle Q\rangle / k_{f} \Delta T / H$ for Rayleigh-Bénard convection can be expressed in terms of $R a_{\lambda_{c}}$ from (7.18) as,

$$
N u=-\frac{5 \times 2^{-14 / 15}}{3} R a_{\lambda_{c}}^{-2 / 15} R a^{1 / 3} \tilde{H}_{1}^{\prime}(0),
$$

where $R a=g \beta \Delta T H^{3} / \nu \alpha$ is the Rayleigh number based on $H$, the distance between the plates, and the temperature difference between the plates, $\Delta T$. The Nusselt number expression shows the $R a^{1 / 3}$ scaling.

RC's asymptotic solutions for $\operatorname{Pr} \rightarrow \infty$ give $\tilde{H}_{1}^{\prime}(0)=-0.4602$. Figure 5 of RC shows the plots of $\tilde{H}_{1}$ vs. $\tilde{\eta}_{1}$ for $P r=5,10$ and $\infty$ and do not show much difference.

\subsubsection{Evaluation of plume spacing}

The mean plume spacing is estimated by equating the Nusselt number expression, (7.19), given by the model, with the Nusselt number correlation of Goldstein et al. (1990) for Rayleigh-Bénard convection at a Prandtl number of 2750 in the Rayleigh number range $10^{9}<R a<10^{14}$. Goldstein et al.'s (1990) results of

$$
S h=0.0659 R a^{1 / 3}
$$

were chosen as $(a)$ the correlation shows the same Rayleigh-number dependence as the model, $(b)$ the Rayleigh number in the present experiments matches their range, and $(c)$ the non-dimensional fluxes $R a_{\delta}^{-1 / 3}$ in Goldstein et al.'s and in the present experiments are similar (cf. figure 4), even if the Prandtl numbers are different.

Equating (7.20) with (7.19), gives the non-dimensional plume spacing as

$$
R a_{\lambda_{c}}^{1 / 3}=\lambda_{c} / Z_{w}=91.7 \simeq 92 .
$$

Equation (7.21) shows that the non-dimensional mean plume spacing is a constant in turbulent natural convection over horizontal surfaces. Higher driving potentials, resulting in higher flux, give rise to lower mean plume spacing so that $\lambda \Delta T_{w}^{1 / 3}$ is a constant for a given fluid, or $\lambda / H \simeq 116 R a^{-1 / 3}$. The mean plume spacing calculated from (7.21) is plotted with the experimental measurements in figure 12(a) and shows satisfactory agreement.

An alternative normalized plume spacing can be obtained as $\lambda_{c} /\left(\alpha \nu k_{f} / g \beta Q\right)^{1 / 4}=$ $R a_{q_{i c}}^{1 / 4}$, where the flux Rayleigh number based on plume spacing is

$$
R a_{q_{\lambda_{c}}}=\frac{g \beta Q \lambda_{c}^{4}}{\alpha \nu k_{f}} .
$$

Note that $\left(\alpha^{3} / g \beta q\right)^{1 / 4}$ is the Townsend's (1959) near-wall length scale for turbulent convection. Using (7.18) for the flux in (7.22) and the value of $R a_{\lambda_{c}}^{1 / 3}$ from (7.21), the plume spacing, normalized by the flux length scale, can be expressed as

$$
R a_{q_{\lambda_{c}}}^{1 / 4}=\frac{\lambda_{c}}{Z_{o} S c^{1 / 4}}=58.54 \simeq 59 .
$$

Figure 18 shows the measured mean plume spacings from the experimental planform images along with the theoretical relation of (7.23). The agreement is satisfactory. 


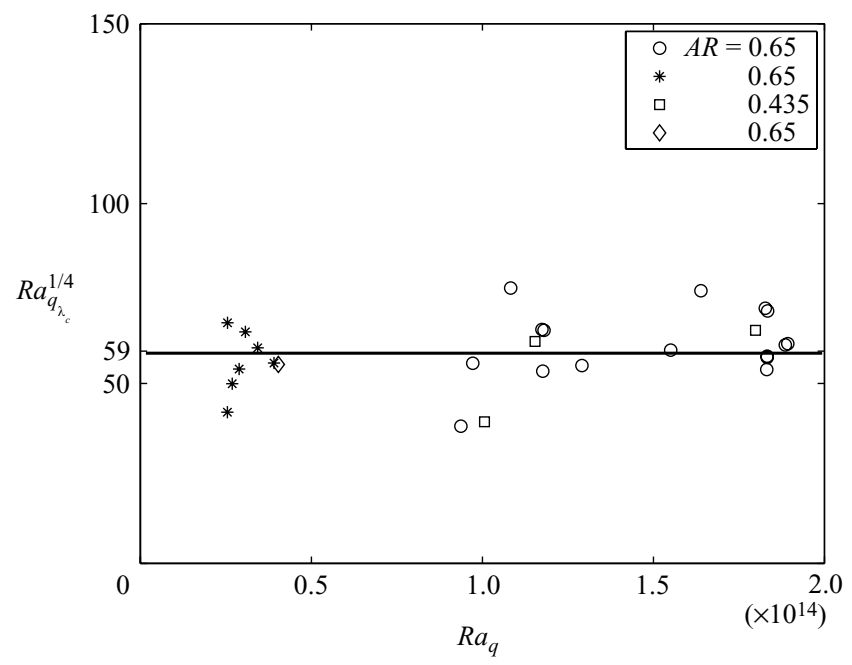

FIGURE 18. Variation of the normalized mean plume spacing, $R a_{q_{\lambda_{c}}^{1 / 4}}$, with flux Rayleigh number.

Therefore, higher flux results in more closely spaced plumes so that $\lambda_{c} q^{1 / 4}$ is a constant for a given fluid.

The model and the spacing statistics specify the plume structure at different $R a_{w}$. As the ratio of $\overline{\ln (\lambda / \bar{\lambda})}$ to $\sigma_{\ln (\lambda / \bar{\lambda})}$ is about 0.325 from figure 16 , figure 15 and figure $16(b)$ specify the distribution of $\lambda / \bar{\lambda}$, as a function of $R a_{w}$. Hence, using the variation of $\bar{\lambda}$ with $R a_{w}$ from (7.21), a complete description of the plume structure at different $R a_{w}$ can be obtained. This knowledge of the near-wall plume structure at different $R a_{w}$ could be used to build wall functions for turbulence modelling of high-Rayleighnumber convection.

The non-dimensional plume spacing in the present high $\operatorname{Pr}$ case is about double the value of $R a_{\lambda_{c}}^{1 / 3}=52$ at $P r=1$ (Theerthan \& Arakeri 1998, 2000). The larger nondimensional plume spacing in high-Prandtl-number convection is an outcome of the gravitational instability condition being satisfied by the thinner thermal boundary layer.

Hence, we have seen that the mean plume spacings predicted from a model, built on the assumption that the near-wall local boundary layers on either side of the sheet plumes in high $R a-S c$ turbulent free convection are laminar natural-convection boundary layers, match the experimental measurements. Many of the earlier boundarylayer measurements can be interpreted based on the above phenomenology. The maximum of horizontal r.m.s. velocities observed at near the thermal boundary-layer edge has been reproduced by Theerthan \& Arakeri (1998) using a similar model at $\operatorname{Pr} \sim 1$. This is expected to be because the maximum horizontal velocities in laminar natural-convection boundary layers occur at around that region owing to the balance of driving horizontal pressure gradient and the viscous resistance. Lui \& Xia (1998) observe non-repeatability of the spatial dependence of thermal boundary-layer thickness, as measured by a single probe, at lower Rayleigh number. We expect this to be due to the presence of laterally moving near-wall sheet plumes. They notice repeatability of an M-shaped spatial dependence of $\delta_{t}$ at higher Rayleigh number, which could be due to the alignment of the sheet plumes owing to the large-scale flow. 
We have considered the local boundary layers associated with each plume. In addition, in the presence of a mean wind, a global boundary layer spanning the width of each circulation cell would exist. If a single circulation cell exists, this global boundary layer would span the width of the experimental cell, as in the theories of Castaing et al. (1989); Siggia (1994) and Grossman \& Lohse (2000). This global boundary layer associated with each circulation cell will be of a mixed convection type. To take into account this effect of the wind, the above model has to be modified to include this second global boundary layer. The modification by the mean wind could be a changed plume spacing and a different local boundary-layer growth. Measurements over a heated flat plate in the presence of a weak wind by Theerthan \& Arakeri (2000) and those over inclined plates by Sparrow \& Husar (1969) show aligned sheet plumes and marginal change in the heat flux due to the wind. However, the effect of mean wind on the plume spacing has not been investigated in earlier mixed convection studies (Maughan \& Incropera 1990; Moharreri, Armaly \& Chen 1988).

Measurements of mean wind strength plume spacing and flux in experiments covering a wide range of Rayleigh number would provide this information.

\section{Conclusions}

In this paper, using a new method of driving the convection by concentration differences across a fine membrane of $0.45 \mu \mathrm{m}$ mean pore size, we were able to study the near-wall structures and their interaction with the large-scale flow in turbulent free convection at $R a_{w} \sim 10^{10}-10^{11}$ at $S c \sim 602$. The flux scaled approximately as the $4 / 3 \mathrm{rd}$ power of effective concentration difference on one side of the membrane, similar to the earlier studies of Rayleigh-Bénard convection at similar Prandtl numbers. Visualizations showed that the near-wall coherent structures in high-Rayleigh-number turbulent free convection are sheet plumes. Depending on the Rayleigh number and the aspect ratio, different types of large-scale flow cells which were driven by plume columns were observed. Multiple large-scale flow cells were observed for $A R=0.65$ and single cells for $A R=0.435$. The large-scale flow creates a near-wall mean shear, which was seen to vary across the cross-section. The orientation of the large-scale flow was seen to change at a time scale much larger than the time scale of one large-scale circulation.

The near-wall flow consists of sheet plumes; the main effect of the large-scale circulation is to align the sheet plumes in the direction of the wind. The plumes are initiated as points and then become elongated along the mean shear direction in areas of larger mean shear. In areas of low mean shear, the plumes are initiated as points but become elongated in directions decided by the flow induced by the adjacent plumes. The effect of the near-wall mean shear was to align the plumes and reduce their lateral movement and merging. The time scale for the merger of the near-wall sheet plumes was an order smaller than the time scale of one large-scale circulation. With increase in Rayleigh number, the number of sheet plumes increases; the plumes become more closely and regularly spaced. The probability distribution function of the plume spacings showed a common log-normal form for all the Rayleigh numbers and the aspect ratios investigated.

We proposed that the near-wall boundary layers in high-Rayleigh-number turbulent free convection are laminar natural-convection boundary layers. The above proposition was verified by a near-wall model, similar to that proposed by Theerthan \& Arakeri (1998), based on the similarity solutions of laminar naturalconvection boundary-layer equations as $\operatorname{Pr} \rightarrow \infty$. The model predicted the nondimensional mean plume spacing as $R a_{\lambda}^{1 / 3}=\lambda_{c} / Z_{w}=91.7$, where $Z_{w}$ is the near-wall 
length scale (equation (1.3)) in turbulent natural convection; the prediction matched the experimental measurements. This implies that in high-Rayleigh-number turbulentfree-convection, higher driving potentials give rise to lower mean plume spacing so that $\lambda \Delta T^{1 / 3}$ or $\lambda q^{1 / 4}$ is a constant for a given fluid.

We have neglected the effect of mean wind in the near-wall model proposed in this paper. It was observed that the mean shear aligns the near-wall sheet plumes. Hence, we could expect that in the presence of mean wind, the boundary layers between the plume sheets would be forced by an external shear. In this case, the nature of the near-wall global boundary layer could be expected to be that of mixed convection. The current analysis must be extended considering mixed convection boundary layers to see whether the anomalous flux scaling in high-Rayleigh-number turbulent-free convection in the presence of a mean wind can be explained from this phenomenology.

\section{REFERENCES}

Adrian, R. J., Ferreira, R. T. D. S. \& Boberg, T. 1986 Turbulent thermal convection in wide horizontal fluid layers. Exps. Fluids 4, 121-141.

Ashrenazi, S. \& Steinberg, V. 1999 High Rayleigh number turbulent convection in a gas near the gas-liquid critical point. Phys. Rev. Lett. 83, 3641-3644.

Belmonte, A., Tilgner, A. \& Libchaber, A. 1994 Temperature and velocity boundary layers in turbulent convection. Phys. Rev. E 50, 269-279.

Burr, U., Kinzelbach, W. \& Tsinober, A. 2003 Is the turbulent wind in convective flows driven by fluctuations? Phys. Fluids 15, 2313-2320.

Castaing, B., Gunaratne, G., Heslot, F., Kadanoff, L., Libchaber, A., Thomae, S., Wu, X., ZALESKI, S. \& ZANETTI, G. 1989 Scaling of hard thermal turbulence in Rayleigh-Bernard convection. J. Fluid. Mech. 204, 1-30.

Chavanne, X., Chilla, F., Chabaud, B., Castaing, B. \& Hebral, B. 2001 Turbulent RayleighBénard convection in gaseous and liquid He. Phys. Fluids 13, 1300-1320.

DAYA, Z. \& ECKE, R. 2001 Does turbulent convection feel the shape of the container. Phys. Rev. Lett. 87, 184501.

DeArdorfF, J. W. 1970 Convective velocity and temperature scales for the unstable planetary boundary layer and for Rayleigh convection. J. Atmos. Sci. 27, 1211-1213.

Frank, H. \& Althoen, S. C. 2002 Statistics, Concepts and Applications. Cambridge University Press.

Funfschilling, D. \& Ahlers, G. 2004 Plume motion and large scale circulation in a cylindrical Rayleigh-Bénard cell. Phys. Rev. Lett. 92, 194502.

Gendrich, C. P. \& Koochesfahani, M. M. 1996 A spatial correlation technique for estimating velocity fields using molecular tagging velocimetry. Exps. Fluids 22, 67-77.

Goldstein, R. J., Chiang, H. D. \& See, D. L. 1990 High-Rayleigh-number convection in a horizontal enclosure. J. Fluid. Mech. 213, 111-126.

Grossman, S. \& Lohse, D. 2000 Scaling in thermal convection: a unifying theory. J. Fluid. Mech. 407, 27.

Grossmann, S. \& Lohse, D. 2001 Thermal convection for large Prandtl numbers. Phys. Rev. Lett. 86, 3316-3319.

Grossman, S. \& LoHSE, D. 2002 Prandtl and Rayleigh number dependence of the Reynolds number in turbulent thermal convection. Phys. Rev. E 66, 016305.

Grossman, S. \& LoHSE, D. 2004 Fluctuations in turbulent Rayleigh-Bénard convection: the role of plumes. Phys. Fluids 16, 4462-4472.

Howard, L. 1964 Convection at high Rayleigh number. In Proc. 11th Intl Congr. Appl. Mech. Munich (ed. Gortler H.), pp. 1109-1115.

Kadanoff, L. P. 2001 Turbulent heat flow: structures and scaling. Phys. Today 54, 34-39.

Kerr, R. M. 1996 Rayleigh number scaling in numerical convection. J. Fluid. Mech. 310, 139-179.

Kerr, R. M. \& Herring, J. R. 2000 Prandtl number dependence of Nusselt number in direct numerical simulations. J. Fluid. Mech. 419, 325-344. 
Kitamura, K. \& Kimura, F. 1995 Heat transfer and fluid flow of natural convection adjacent to upward facing plates. Intl J. Heat Mass Transfer 38, 3149-3159.

Kraichnan, R. H. 1962 Turbulent thermal convection at arbitrary Prandtl numbers. Phys. Fluids 5, 1374-1389.

Krishnamurthi, R. 1970 On the transition to turbulent convection. J. Fluid. Mech. 42, 295-320.

Lam, S., Shang, X. D., Zhou, S. Q. \& XIA, K. Q. 2002 Prandtl number dependence of the viscous boundary layer and the Reynolds numbers in Rayleigh number convection. Phys. Rev. E 65, 066306.

LIDE, D. R. (ed.) 2001 CRC Handbook of Chemistry and Physics, 80th edn. CRC Press.

LUI, S. L. \& XIA, K. Q. 1998 Spatial structure of the thermal boundary layer in turbulent convection. Phys. Rev. E 57, 5494.

Maughan, J. R. \& InCROPERA, F. P. 1990 Regions of heat transfer enhancement for laminar mixed convection in a parallel plate channel. Intl J. Heat Mass Transfer 33, 555-570.

Moharreri, S. S., Armaly, B. F. \& Chen, T. S. 1988 Measurements in the transition vortex flow regime of mixed convection above a horizontal heated plate. Trans. ASME, J. Heat Transfer 110, 358-364.

Niemela, J. J., Skrbek, L., Sreenivasan, K. R. \& Donelly, R. J. 2000 Turbulent convection at very high Rayleigh numbers. Nature 404, 837-840.

Niemela, J. J., Skrbek, L., Sreenivasan, K. R. \& Donnely, R. J. 2001 The wind in confined thermal convection. J. Fluid. Mech. 449, 169-178.

Niemela, J. J. \& Sreenivasan, K. R. 2003 Confined turbulent convection. J. Fluid. Mech. 481, 355-384.

ORION 1999a ORION, Sensing the Future, 1999 Laboratory Products and Electro Chemistry Handbook. ORION Research.

ORION 1999 b Sensorlink ${ }^{T M}$ Model PCM100 Instruction Manual. ORION Research.

PuthenveEtTiL, B. A. 2004 Investigations on high Rayleigh number turbulent free-convetion. PhD thesis, Indian Institute of Science, Bangalore, http://etd.ncsi.iisc.ernet.in

Puthenveettil, B. A. \& Arakeri, J. H. 2005 Multifractal nature of plume structure in high Rayleigh number convection. J. Fluid. Mech. 526, 245-256.

Rotem, Z. \& Classen, L. 1969 Natural convection above unconfined horizontal surfaces. J. Fluid. Mech. 39, 173-192.

Schmidt, H. \& Schumann, U. 1989 Coherent structure of the convective boundary layer derived from large-eddy simulations. J. Fluid. Mech. 200, 541-562.

Shraiman, B. I. \& Siggia, E. D. 1990 Heat transport in high Rayleigh number convection. Phys. Rev. A 42, 3650-3653.

Siggia, E. D. 1994 High Rayleigh number convection. Annu. Rev. Fluid Mech. 26, 137-168.

Spangenberg, W. G. \& Rowland, W. G. 1961 Convective circulation in water induced by evaporative cooling. Phys. Fluids 4, 743-750.

Sparrow, E. \& Husar, R. 1969 Longitudinal vortices in natural convection flow on inclined plates. J. Fluid. Mech. 37, 251-255.

Spiegel, E. A. 1971 Convection in stars i. Basic Boussinesque convection. Annu. Rev. Astron. Astrophys. 9 323-352.

Theerthan, S. A. \& Arakeri, J. H. 1994 Planform structure of turbulent Rayleigh-Bénard convection. Intl Commun. Heat Mass Transfer 21, 561-572.

Theerthan, S. A. \& Arakeri, J. H. 1998 A model for near wall dynamics in turbulent RayleighBénard convection. J. Fluid. Mech. 373, 221-254.

Theerthan, S. A. \& Arakeri, J. H. 2000 Plan form structure and heat transfer in turbulent free convection over horizontal surfaces. Phys. Fluids 12, 884-894.

TownsEnd, A. A. 1959 Temperature fluctuations over a heated horizontal surface. J. Fluid. Mech. 5, 209-211.

Verzicco, R. \& CAMussi, R. 1999 Prandtl number effects in convective turbulence. J. Fluid. Mech. 383, 55-73.

Verzicco, R. \& CAmussi, R. 2003 Numerical experiments on strongly turbulent thermal convection in a slender cylindrical cell. J. Fluid. Mech. 477, 19-49.

Werne, J. 1993 Structure of hard - turbulent convection in two dimensions: numerical evidence. Phys. Rev. E 48, 1020-1035. 
Wu, X. \& Libchaber, A. 1992 Scaling relations in thermal turbulence: the aspect ratio dependence. Phys. Rev. A 45, 842-845.

XIA, K. Q., LAM, S. \& Zhou, S. Q. 2002 Heat flux measurement in high Prandtl number turbulent Rayleigh-Bénard convection. Phys. Rev. Lett. 88, 064501.

XIA, K. Q., Sun, C. \& Zhou, S. Q. 2003 Particle image velocimetry measurement of the velocity field in turbulent thermal convection. Phys. Rev. E 68, 066303.

XIN, Y. B. \& XIA, K. Q. 1997 Boundary layer length scales in convective turbulence. Phys. Rev. E 56, 3010.

Zocchi, G., Moses, E. \& Libchaber, A. 1990 Coherent structures in turbulent convection, an experimental study. Physica A 166, 387-407. 